\title{
HDAC inhibitors induce tumor-cell-selective pro-apoptotic transcriptional responses
}

\author{
JE Bolden ${ }^{1}$, W Shi ${ }^{2,3}$, K Jankowski $^{4}$, C-Y Kan ${ }^{4}$, L Cluse ${ }^{1,5}$, BP Martin ${ }^{1,5}$, KL MacKenzie $^{4}$, GK Smyth ${ }^{2,6}$ and RW Johnstone ${ }^{*, 1,5}$
}

The identification of recurrent somatic mutations in genes encoding epigenetic enzymes has provided a strong rationale for the development of compounds that target the epigenome for the treatment of cancer. This notion is supported by biochemical studies demonstrating aberrant recruitment of epigenetic enzymes such as histone deacetylases (HDACs) and histone methyltransferases to promoter regions through association with oncogenic fusion proteins such as PML-RAR $\alpha$ and AML1-ETO. HDAC inhibitors (HDACi) are potent inducers of tumor cell apoptosis; however, it remains unclear why tumor cells are more sensitive to HDACi-induced cell death than normal cells. Herein, we assessed the biological and molecular responses of isogenic normal and transformed cells to the FDA-approved HDACi vorinostat and romidepsin. Both HDACi selectively killed cells of diverse tissue origin that had been transformed through the serial introduction of different oncogenes. Time-course microarray expression profiling revealed that normal and transformed cells transcriptionally responded to vorinostat treatment. Over 4200 genes responded differently to vorinostat in normal and transformed cells and gene ontology and pathway analyses identified a tumor-cell-selective pro-apoptotic gene-expression signature that consisted of BCL2 family genes. In particular, HDACi induced tumor-cell-selective upregulation of the pro-apoptotic gene BMF and downregulation of the pro-survival gene BCL2A1 encoding BFL-1. Maintenance of BFL-1 levels in transformed cells through forced expression conferred vorinostat resistance, indicating that specific and selective engagement of the intrinsic apoptotic pathway underlies the tumor-cell-selective apoptotic activities of these agents. The ability of HDACi to affect the growth and survival of tumor cells whilst leaving normal cells relatively unharmed is fundamental to their successful clinical application. This study provides new insight into the transcriptional effects of HDACi in human donor-matched normal and transformed cells, and implicates specific molecules and pathways in the tumorselective cytotoxic activity of these compounds.

Cell Death and Disease (2013) 4, e519; doi:10.1038/cddis.2013.9; published online 28 February 2013

Subject Category: Cancer

Deregulation of histone deacetylase (HDAC) activity in tumors, through over-expression of individual HDACs, ${ }^{1-8}$ or through aberrant HDAC recruitment to promoter regions through association with oncogenic fusion proteins such as PML-RAR $\alpha^{9,10}$ and AML1-ETO ${ }^{11,12}$ has provided a strong rationale for the clinical application of HDAC inhibitors (HDACi). HDACi can induce cell-cycle arrest, differentiation and cell death in vitro and mediate tumor-cell-selective apoptosis at drug concentrations that leave normal cells relatively unharmed. ${ }^{13-15}$ We previously demonstrated that apoptotic sensitivity of tumor cells to HDACi in vitro correlated with therapeutic responsiveness in vivo. ${ }^{16,17}$ However, it is not clear why tumor cells are relatively hypersensitive to HDACimediated apoptosis and normal cells are relatively resistant.

To assess the tumor-specific molecular and biological activities of HDACi, we used a defined model of human cell transformation in which normal cells had been converted to transformed (tumorigenic) states through the sequential introduction of genes encoding the SV40 large T and small $t$ antigens, hTERT and H-RAS ${ }^{\mathrm{G} 12 \mathrm{~V}}$. $^{18,19}$ The HDACi vorinostat and romidepsin induced apoptosis in a tumor-cell-selective manner. Time-course gene expression profiling revealed that normal and transformed cells transcriptionally responded to HDACi treatment; however, 4200 genes responded differently to vorinostat treatment in normal and transformed cells. Gene ontology (GO) and pathway analyses identified a tumor-cellselective pro-apoptotic gene-expression signature containing effectors of the intrinsic apoptotic pathway. Regulation of a pro-apoptotic BCL2-family gene signature correlated with the relative sensitivity of transformed cells to HDACi-mediated apoptosis. Furthermore, a role for HDACi-mediated repression of $B C L 2 A 1$ in the induction of tumor cell apoptosis was confirmed, and we formally demonstrated that forced expression of BFL-1 encoded by BCL2A1 suppressed the apoptotic

\footnotetext{
${ }^{1}$ Cancer Therapeutics Program, Gene Regulation Laboratory, The Peter MacCallum Cancer Centre, St. Andrews Place, East Melbourne 3002, Victoria, Australia; ${ }^{2}$ Walter and Eliza Hall Institute of Medical Research, Royal Parade, Parkville 3052, Victoria, Australia; ${ }^{3}$ Department of Computing and Information Systems, University of Melbourne, Parkville 3010, Victoria, Australia; ${ }^{4}$ Cancer Cell Development Group, Children's Cancer Institute Australia, Lowy Cancer Centre, University of New South Wales, Sydney 2052, New South Wales, Australia; ${ }^{5}$ Sir Peter MacCallum Department of Oncology, University of Melbourne, Parkville 3010, Victoria, Australia and ${ }^{6}$ Department of Mathematics and Statistics, University of Melbourne, Parkville 3010, Victoria, Australia

*Corresponding author: RW Johnstone, Gene Regulation Laboratory, Peter MacCallum Cancer Centre, St. Andrews Place, A'Beckett St., East Melbourne, Victoria 3002, Australia. Tel: + 6139656 3727; Fax: + 6139656 1411; E-mail: ricky.johnstone@ @etermac.org

Keywords: epigenetics; apoptosis; tumor-selective; microarray

Abbreviations: HDAC, histone deacetylase; BMEC, bone marrow endothelial cell; HDACi, histone deacetylase inhibitor; $\mathrm{CHX}$, cycloheximide; PI, propidium iodide Received 18.12.12; accepted 27.12.12; Edited by G Melino
} 
effects of vorinostat in transformed BJ fibroblasts. Collectively, these data enhance our understanding of the molecular consequences of HDAC inhibition, and provide a mechanistic basis for the tumor-selective biological effects of these agents.

\section{Results}

HDAC inhibitors selectively kill tumor cells. Matched normal (BJ) and transformed (BJ LTSTERas) fibroblasts were treated with vorinostat over $72 \mathrm{~h}$, and cell death was analyzed (Figures $1 \mathrm{a}$ and $\mathrm{b}$ ). Following $24 \mathrm{~h}$ vorinostat treatment, there was a marginal increase in death of transformed BJ LTSTERas fibroblasts that increased substantially following extended drug exposure. BJ LTSTERas fibroblasts were significantly more sensitive to vorinostat than BJ cells (Figures $1 \mathrm{a}$ and b). Vorinostat induced comparable time-dependent hyperacetylation of histone $\mathrm{H} 3$ (Figure 1c) and $\alpha$-tubulin (Figure 1d) in both cell types, suggesting that the relative resistance of normal cells to vorinostat-mediated apoptosis was not due to a failure of vorinostat to inhibit HDACs in these cells. We next tested the sensitivity of BJ and BJ LTSTERas fibroblasts to romidepsin, an HDACi that selectively inhibits class I HDACs. ${ }^{20-22}$ BJ LTSTERas fibroblasts were more sensitive than BJ cells to romidepsin-induced apoptosis, even though there was equivalent acetylation of histone $\mathrm{H} 3$ in romidepsin-treated BJ and BJ LSTERas fibroblasts (Figures $1 e$ and f). As $\alpha$-tubulin is deacetylated by HDAC6 that is not effectively inhibited by romidepsin, romidepsin did not induce hyperacetylation of $\alpha$-tubulin.

The sensitivity of donor-matched normal and transformed bone marrow endothelial cells (BMECs) to HDACi was also assessed. BMEC and BMEC LTSTERas were treated with vorinostat for $48 \mathrm{~h}$ and analyzed for annexin- $\mathrm{V}$ binding and propidium iodide uptake (Supplementary Figure 1A). Consistent with observations in the BJ fibroblast system, transformed BMEC were comparatively hypersensitive to vorinostat-mediated death.

Finally, we determined whether the mechanisms by which normal cells were transformed affected the relative sensitivity of cells to vorinostat-induced apoptosis. Transformed BJ fibroblasts in which SV40 large T antigen was functionally replaced with HPV16 $\mathrm{E} 6$ and $\mathrm{E} 7,{ }^{23}$ were tested for vorinostatsensitivity. BJ cells expressing hTERT, E6, E7, H-RAS ${ }^{\mathrm{G} 12 \mathrm{~V}}$ and ST (BJ E6E7 TRST) were sensitive to vorinostat relative to normal BJ fibroblasts when measured over a 72-h time course (Supplementary Figure 1B). These data demonstrated that HDACis selectively killed transformed cells while mediating equivalent histone hyperacetylation in normal and transformed cells.

Vorinostat induces tumor-cell-selective gene expression changes. We hypothesized that tumor-cell-selective gene expression changes underpinned the differential sensitivity of isogenic tumor and normal cells to HDACi-induced apoptosis. To investigate whether de novo protein synthesis was required for HDACi-induced death, BJ LTSTERas fibroblasts were pre-treated for $1 \mathrm{~h}$ with cycloheximide $(\mathrm{CHX})$ before the addition of vorinostat. $\mathrm{CHX}$ treatment significantly inhibited vorinostat-mediated apoptosis after $48 \mathrm{~h}$ of drug treatment (Figures $2 \mathrm{a}$ and b). Given the requirement of de novo protein expression for the induction of apoptosis by vorinostat, a time-course microarray study was conducted. An early (4h) and intermediate $(12 \mathrm{~h})$ time point was selected for the microarray study on the basis of candidate quantitative realtime polymerase chain reaction (qRT-PCR) analyses of CDKN1A (Figures 2c and d), a gene commonly induced by $\mathrm{HDACi}^{20,24} C D K N 1 A$ mRNA was induced by vorinostat in BJ and BJ LTSTERas cells; however, the magnitude of induction was greater in transformed cells (Figures $2 \mathrm{c}$ and d). The abundance of CDKN1A mRNA in BJ and BJ LTSTERas cells after $24 \mathrm{~h}$ of vorinostat treatment was similar, as the threshold cycle $(\mathrm{Ct})$ values relative to the control gene RPL32 were similar in both cell types (data not shown). The hyper-induction of CDKN1A in BJ LTSTERas fibroblasts over time reflects the lower basal expression in these cells (at time $0 \mathrm{~h}$ ).

Vorinostat-induced changes in gene expression in BJ and BJLSTRas cells. Affimetrix microarray assays showed that the number of vorinostat-regulated probe sets increased in both cell types as a function of time; however, the overall response in BJ LTSTERas cells consistently exceeded that of BJ cells by $\sim 1000$ probe sets at every time point (Figure 3a). At the time of onset of apoptosis $(24 \mathrm{~h})$, the number of vorinostat-regulated probe sets in BJ and BJ LTSTERas cells corresponded to 21.1 and $23.0 \%$ of the total number of probe sets represented on the arrays, respectively. Collectively, these data indicated that both BJ and BJ LTSTERas fibroblasts were capable of mounting a transcriptional response to vorinostat although

\footnotetext{
Figure 1 Matched normal and tumor cells are differentially sensitive to vorinostat-mediated apoptosis. (a, b) BJ and BJ LTSTERas cells were treated with $0,2.5,5,10,25$ or $50 \mu \mathrm{M}$ doses of vorinostat for 24,48 or $72 \mathrm{~h}$. Following treatment, all cells were harvested and (a) stained for annexin-V-APC binding and propidium iodide uptake, and analyzed by flow cytometry; (b) fixed with $50 \%$ (v/v) ethanol in PBS, stained with propidium iodide and analyzed by flow cytometry. The percentage of annexin-V-APC, propidium iodide double positive cells or cells with sub-diploid DNA content is shown in panels $(a, b)$, respectively. Data are presented as mean \pm standard error of at least three independent experiments. $P$-values indicate statistical significance of cell type and dose response interactions as measured by two-way ANOVA, where $P<0.05$ is considered to be significant. (c, d) BJ (top panels) and BJ LTSTERas (bottom panels) cells were treated with $25 \mu \mathrm{M}$ vorinostat or DMSO equivalent for 0-32 h. Protein extracts were analyzed by western blotting for: (c) acetylation of histone $\mathrm{H} 3$ with a pan-acetylated Histone $\mathrm{H} 3$ antibody, expression of $\alpha$-Tubulin was used as a loading control; (d) acetylation of $\alpha$-Tubulin with an anti-acetylated tubulin antibody, expression of $\beta$-Actin served as loading control. Western blots shown are representative of two independent experiments. (e) BJ and BJ LTSTERas were treated with $0,2.5,5,10,25$ and $50 \mathrm{nM}$ concentrations of romidepsin for $48 \mathrm{~h}$. Following treatment, all cells were harvested and stained for annexin-V-APC binding and propidium iodide uptake (top panel) and analyzed by flow cytometry or fixed with $50 \%$ (v/v) ethanol in PBS, stained with propidium iodide (bottom panel) and analyzed by flow cytometry. The percentage of annexin-V-APC, propidium iodide double positive cells or cells with sub-diploid DNA content is shown. Data are presented as mean \pm standard error of at least five independent experiments. The difference in apoptotic sensitivity of BJ and BJ LTSTERas (interaction between cell type and romidepsin dose) was statistically significant $(P<0.05)$, as determined by two-way ANOVA. (f) BJ and BJ LTSTERas fibroblasts were treated with $50 \mathrm{nM}$ romidepsin for $0-24 \mathrm{~h}$ or with $25 \mu \mathrm{M}$ vorinostat for $6 \mathrm{~h}(\mathrm{~V})$. Immunoblots were probed with antibodies against acetylated histone $\mathrm{H} 3(\mathrm{AcH} 3)$, acetylated $\alpha-T u b u l i n$ (Ac- $\alpha$-Tubulin) and total $\alpha$-Tubulin to control for protein loading. Images are representative of two independent experiments
} 
$\sim 1000$ additional probe sets were significantly regulated by vorinostat in the transformed cells at every time point analyzed.

The Connectivity Map (Cmap) tool ${ }^{25}$ was used to test whether the vorinostat signatures from BJ and BJ LTSTERas fibroblasts were similar to other drug-induced expression profiles. The Cmap compound with highest connectivity to the BJ and BJ LTSTERas profiles generated in this study was vorinostat (Tables 1 and 2). Two hydroxamic-acid based HDACi, trichostatin A (TSA) and scriptaid, also demonstrated
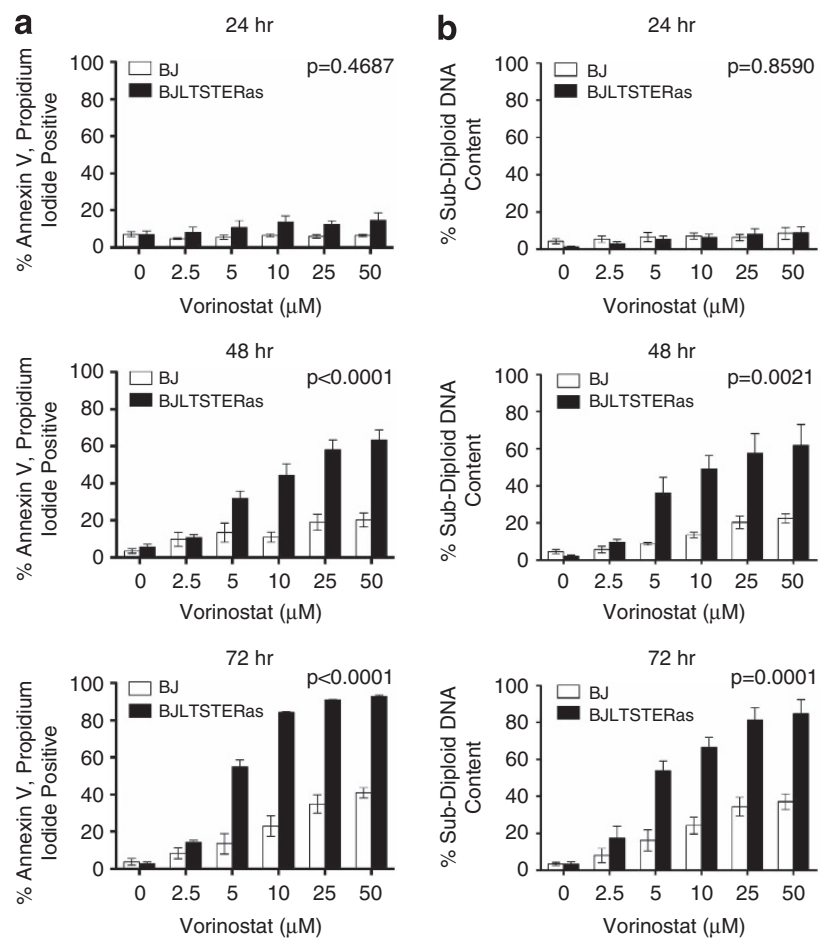

BJ

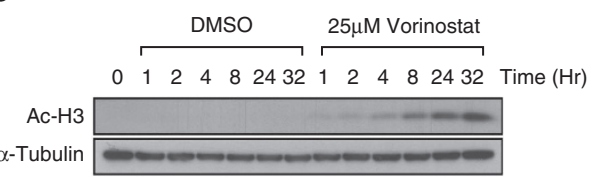

BJ LTSTERas

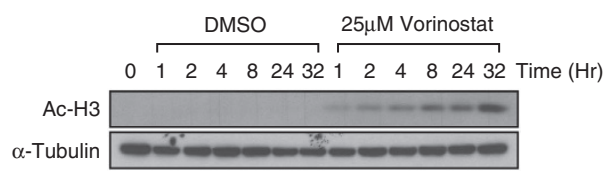

e
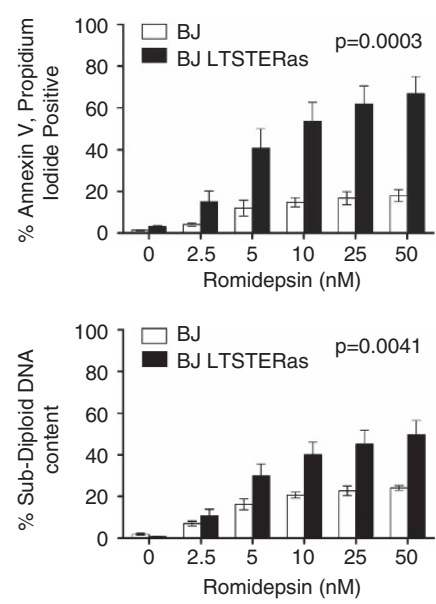

d

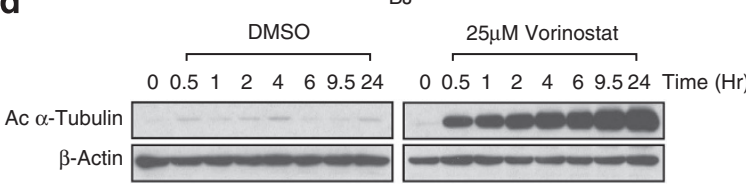

BJ LTSTERas

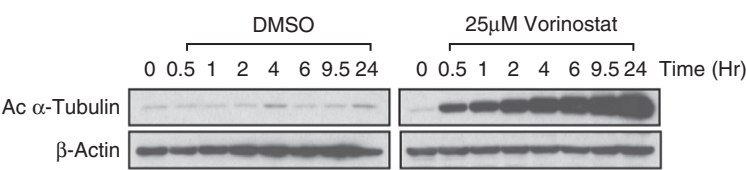

f

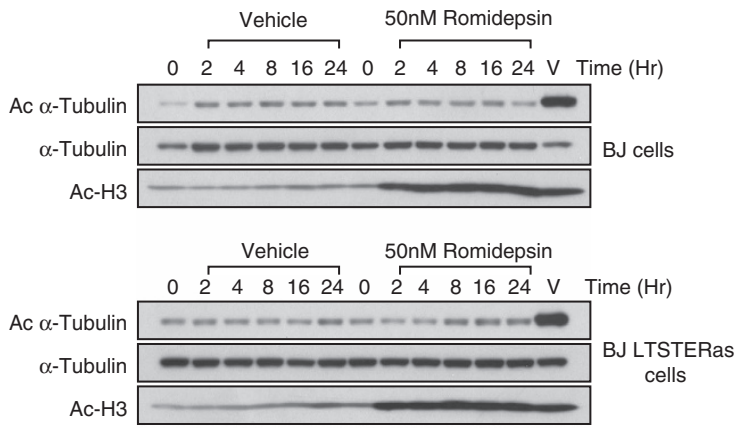



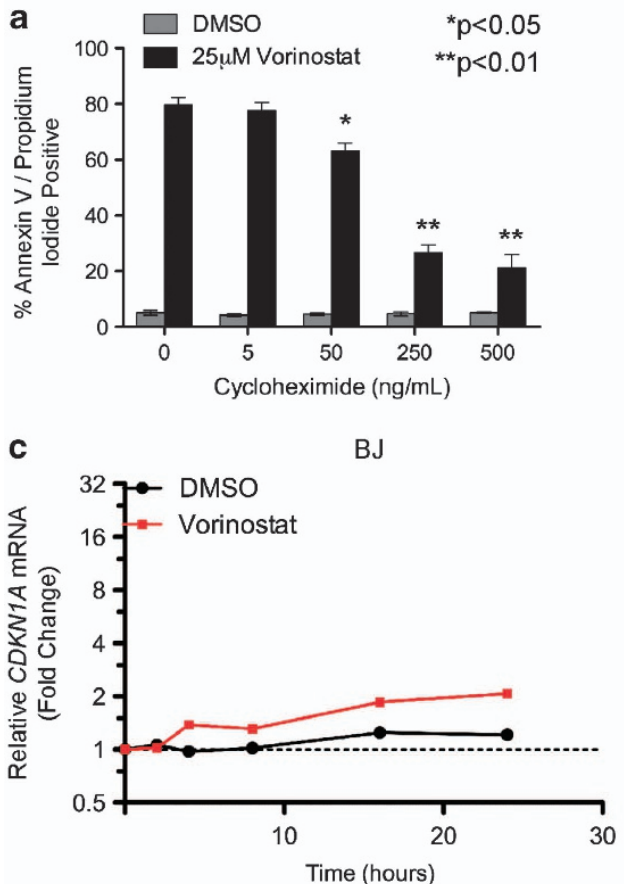

b

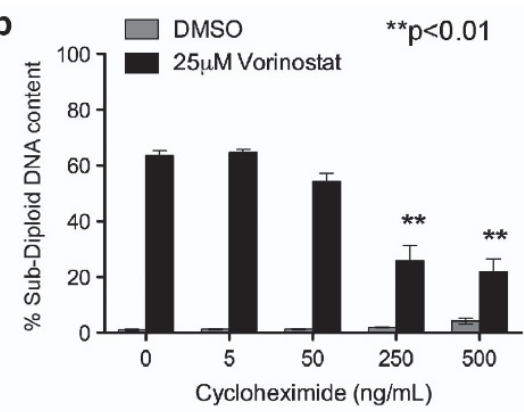

d

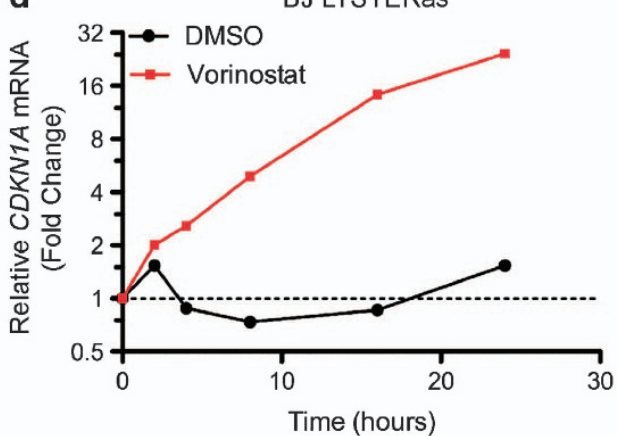

Figure 2 Vorinostat-mediated apoptosis requires de novo protein synthesis. (a, b) BJ and BJ LTSTERas cells were pre-treated with 0, 5, 50, 250 and 500 ng/ml CHX to inhibit new protein synthesis and incubated with $25 \mu \mathrm{M}$ vorinostat for $48 \mathrm{~h}$. Cells were harvested and analyzed for cell death by flow cytometry. (a) Cells were stained with annexin-V-APC and propidium iodide. The percentages of cells staining positively for both markers are shown. (b) Cells were fixed with $50 \%$ (v/v) ethanol in PBS, stained with propidium iodide and analyzed for DNA content. The percentages of cells with sub-2N DNA content are shown. All data are presented as mean \pm S.E.M. of three independent experiments. ${ }^{*} P<0.05,{ }^{* *} P<0.01$ as determined by a two-tailed Student's t-test, comparing $25 \mu \mathrm{M}$ vorinostat treatments in the presence and absence of $\mathrm{CHX}$. (c, d) BJ and BJ LTSTERas cells were treated with $25 \mu \mathrm{M}$ vorinostat (red) or DMSO (black) for $0,2,4,8,16$ and $24 \mathrm{~h}$. Expression of CDKN1A in (c) BJ and (d) BJ LTSTERas cells was analyzed by qRT-PCR. Messenger RNA levels were calculated relative to that of transcripts from the non-HDACi-regulated control gene RPL32. Fold expression changes at each time point are plotted relative to time $0 \mathrm{~h}$ (untreated cells)

a

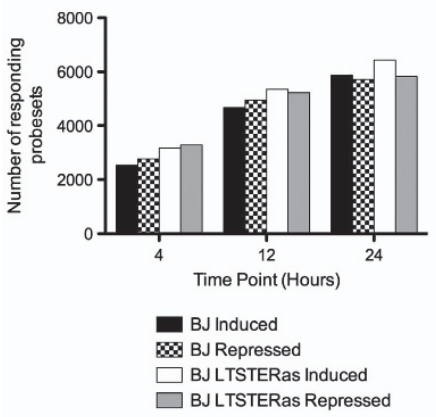

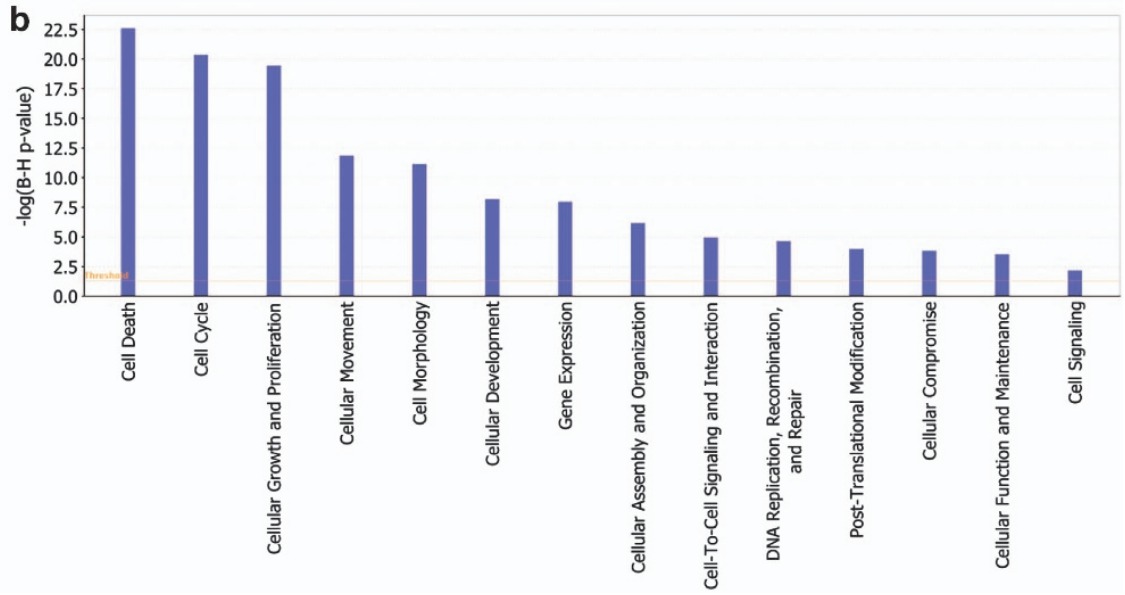

Figure 3 Regulation of gene expression in BJ and BJ LTSTERas by vorinostat. (a) The number of probe sets that were significantly altered in BJ and BJ LTSTERas cells in response to treatment with vorinostat. Probe sets that were induced and repressed by at least 1.5 -fold at any of the 4,12 and $24 \mathrm{~h}$ time points relative to time $0 \mathrm{~h}$ are shown. (b) Genes that responded differently to vorinostat treatment in matched normal and transformed cells (contrast genes) were analyzed using the IPA tool. The associations of various molecular and cellular functions with contrast genes are plotted in decreasing order of statistical significance, according to $-\log 2(P$-value). $P$-values were adjusted for multiple testing using the method of Benjamini and Hochberg. The orange line indicates the threshold for statistical significance $(P<0.05)$

high degrees of connectivity, which indicated that the probe sets regulated by vorinostat in BJ and BJ LTSTERas fibroblasts were similar to those regulated by different $\mathrm{HDACi}$ in diverse cell types.
Identification of genes that respond differently to vorinostat treatment in normal and transformed fibroblasts. We identified probe sets that responded differently to vorinostat treatment in matched normal and 
Table 1 Connectivity map for vorinostat-responding genes in treated BJ cells

\begin{tabular}{|c|c|c|c|c|c|c|c|}
\hline Rank & Cmap name & Mean & $n$ & Enrichment & $P$-value & Specificity & $\%$ Non-null \\
\hline 1 & Vorinostat & 0.762 & 12 & 0.977 & 0.00000 & 0.0151 & 100 \\
\hline 2 & Trichostatin A & 0.695 & 182 & 0.922 & 0.00000 & 0.0047 & 96 \\
\hline 3 & Resveratrol & 0.342 & 9 & 0.873 & 0.00000 & 0.0049 & 100 \\
\hline 4 & Valproic acid & 0.238 & 57 & 0.488 & 0.00000 & 0.0000 & 68 \\
\hline 5 & Sirolimus & 0.102 & 44 & 0.439 & 0.00000 & 0.1024 & 50 \\
\hline 6 & LY-294002 & 0.194 & 61 & 0.421 & 0.00000 & 0.0940 & 67 \\
\hline 7 & Scriptaid & 0.747 & 3 & 0.980 & 0.00004 & 0.0000 & 100 \\
\hline 8 & Rifabutin & 0.695 & 3 & 0.973 & 0.00004 & 0.0000 & 100 \\
\hline 9 & Prochlorperazine & 0.209 & 16 & 0.571 & 0.00004 & 0.0631 & 81 \\
\hline 10 & Trifluoperazine & 0.204 & 16 & 0.549 & 0.00006 & 0.1442 & 68 \\
\hline 11 & Thioridazine & 0.196 & 20 & 0.509 & 0.00008 & 0.2329 & 80 \\
\hline 12 & Syrosingopine & 0.327 & 4 & 0.900 & 0.00010 & 0.0093 & 100 \\
\hline 13 & CP-690334-01 & 0.275 & 8 & 0.685 & 0.00036 & 0.0121 & 75 \\
\hline 14 & Loperamide & 0.235 & 6 & 0.756 & 0.00050 & 0.0152 & 100 \\
\hline 15 & Parbendazole & -0.365 & 4 & -0.874 & 0.00054 & 0.0000 & 50 \\
\hline 16 & Tanespimycin & 0.115 & 62 & 0.248 & 0.00064 & 0.5285 & 54 \\
\hline 17 & Mefloquine & 0.291 & 5 & 0.795 & 0.00084 & 0.0588 & 100 \\
\hline 18 & Fluphenazine & 0.166 & 18 & 0.441 & 0.00089 & 0.1814 & 66 \\
\hline 19 & Securinine & -0.582 & 4 & -0.852 & 0.00092 & 0.0127 & 75 \\
\hline 20 & MS-275 & 0.609 & 2 & 0.974 & 0.00111 & 0.0433 & 100 \\
\hline
\end{tabular}

Table 2 Connectivity map for vorinostat-responding genes in treated BJ LTSTERas cells

\begin{tabular}{|c|c|c|c|c|c|c|c|}
\hline Rank & Cmap name & Mean & $n$ & Enrichment & $P$-value & Specificity & $\%$ Non-null \\
\hline 1 & Vorinostat & 0.816 & 12 & 0.978 & 0.00000 & 0.0151 & 100 \\
\hline 2 & Trichostatin A & 0.703 & 182 & 0.932 & 0.00000 & 0.0000 & 96 \\
\hline 3 & Resveratrol & 0.243 & 9 & 0.861 & 0.00000 & 0.0098 & 100 \\
\hline 4 & CP-690334-01 & 0.358 & 8 & 0.802 & 0.00000 & 0.0121 & 87 \\
\hline 5 & 15-Delta prostaglandin J2 & 0.202 & 15 & 0.696 & 0.00000 & 0.0447 & 86 \\
\hline 6 & LY-294002 & 0.168 & 61 & 0.577 & 0.00000 & 0.0134 & 73 \\
\hline 7 & Valproic acid & 0.171 & 57 & 0.482 & 0.00000 & 0.0000 & 61 \\
\hline 8 & Scriptaid & 0.777 & 3 & 0.978 & 0.00004 & 0.0000 & 100 \\
\hline 9 & Rifabutin & 0.650 & 3 & 0.973 & 0.00010 & 0.0000 & 100 \\
\hline 10 & 5707885 & 0.282 & 4 & 0.899 & 0.00030 & 0.0052 & 100 \\
\hline 11 & Thiostrepton & 0.247 & 4 & 0.880 & 0.00034 & 0.0392 & 100 \\
\hline 12 & Phenoxybenzamine & 0.261 & 4 & 0.876 & 0.00046 & 0.2030 & 100 \\
\hline 13 & Ciclopirox & 0.196 & 4 & 0.864 & 0.00101 & 0.0338 & 100 \\
\hline 14 & Isotretinoin & 0.197 & 4 & 0.842 & 0.00050 & 0.0000 & 100 \\
\hline 15 & Bacitracin & -0.410 & 3 & -0.913 & 0.00112 & 0.0121 & 100 \\
\hline 16 & Sertaconazole & -0.249 & 4 & -0.834 & 0.00137 & 0.0000 & 75 \\
\hline 17 & Mycophenolic acid & 0.252 & 3 & 0.910 & 0.00154 & 0.0452 & 100 \\
\hline 18 & Syrosingopine & 0.260 & 4 & 0.822 & 0.00173 & 0.0326 & 100 \\
\hline 19 & MG-262 & 0.314 & 3 & 0.902 & 0.00184 & 0.1676 & 100 \\
\hline 20 & Fenoprofen & -0.187 & 6 & -0.683 & 0.00266 & 0.0255 & 50 \\
\hline
\end{tabular}

transformed cells (contrast probe sets) at any of the three time points (relative to time $0 \mathrm{~h}$ ), as we hypothesized that contrast gene expression might underpin the different biological responses of donor-matched cells to vorinostat treatment. In total, 5959 contrast probe sets were identified and these were differently regulated by vorinostat in terms of either the direction (induction or repression) or the magnitude (degree of induction or repression) of the vorinostat response in BJ and BJ LTSTERas cells. Of the 5959 contrast probe sets, 2945 were equally expressed in untreated normal and tumor cells. However, 6226 of the 9240 probe sets with basal expression differences did not respond differently to vorinostat in the two cell types. These data indicated that the basal gene expression levels did not determine the capacity of genes to differently respond to vorinostat treatment in BJ and BJ LTSTERas fibroblasts. GO and pathway analyses were performed on the contrast gene list using the Ingenuity
Pathways Analysis (IPA) tool (Figure 3b). 'Cell Death' was the most significantly represented molecular and cellular function associated with contrast genes (false discovery rate $(F D R)=3 \times 10^{-23}$ ) while genes involved 'cell-cycle' $\left(\mathrm{FDR}=5 \times 10^{-21}\right)$ and 'cellular growth and proliferation' $\left(F D R=4 \times 10^{-20}\right)$ were also significantly represented on the contrast list.

We further analyzed the contrast responses, in particular those genes that were selectively or hyper-induced in BJ LTSTERas cells compared with BJ cells, using GO analyses and the Database for Annotation, Visualization and Integrated Discovery (DAVID) tool (Supplementary Tables 1-3). Genes involved in 'induction of programmed cell death' $(P=0.0130)$ and 'apoptotic program' $(P=0.0024)$ were over-represented in vorinostat-treated BJ LSTRas cells compared with BJ cells (Supplementary Tables 1-3). Many genes encoding components of the intrinsic and extrinsic apoptotic pathway were 
a

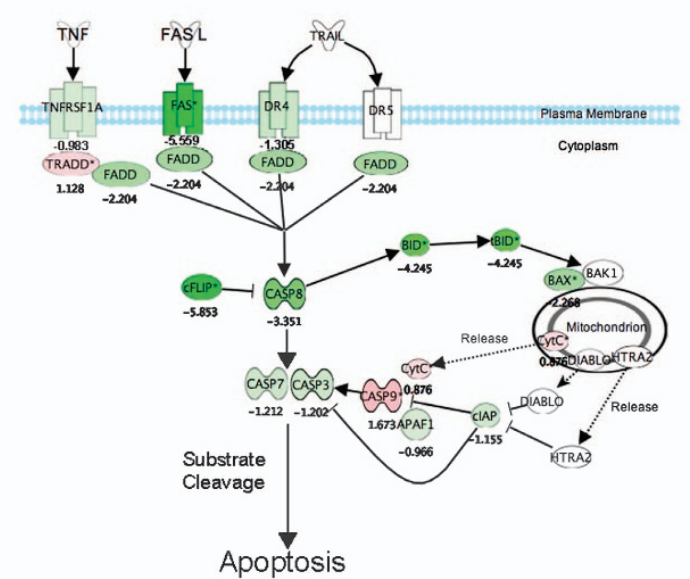

b

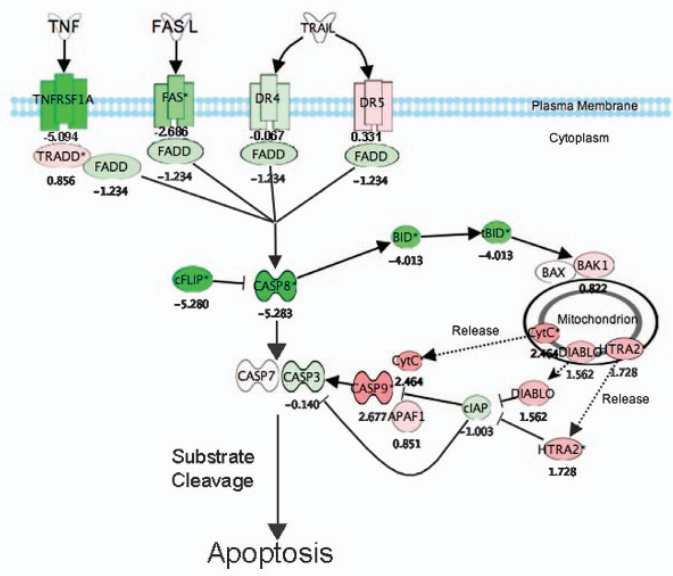

d
C

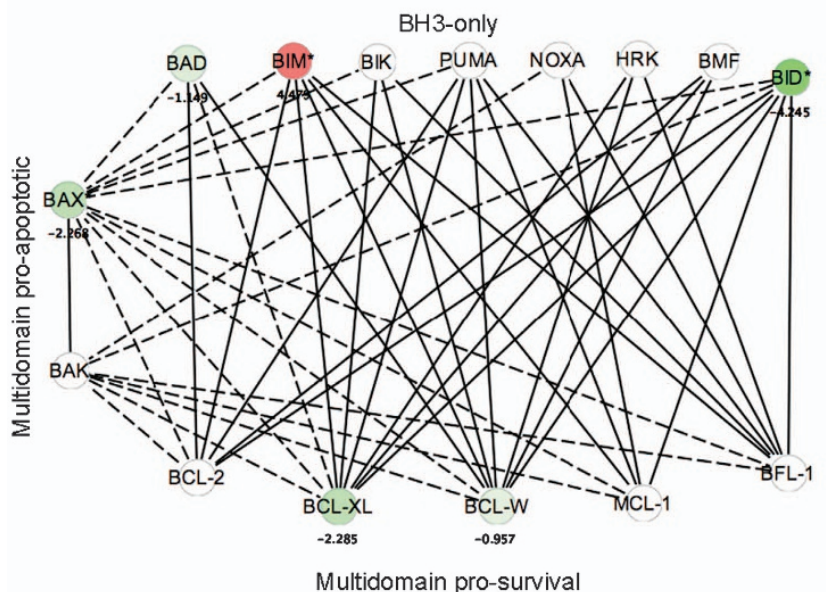

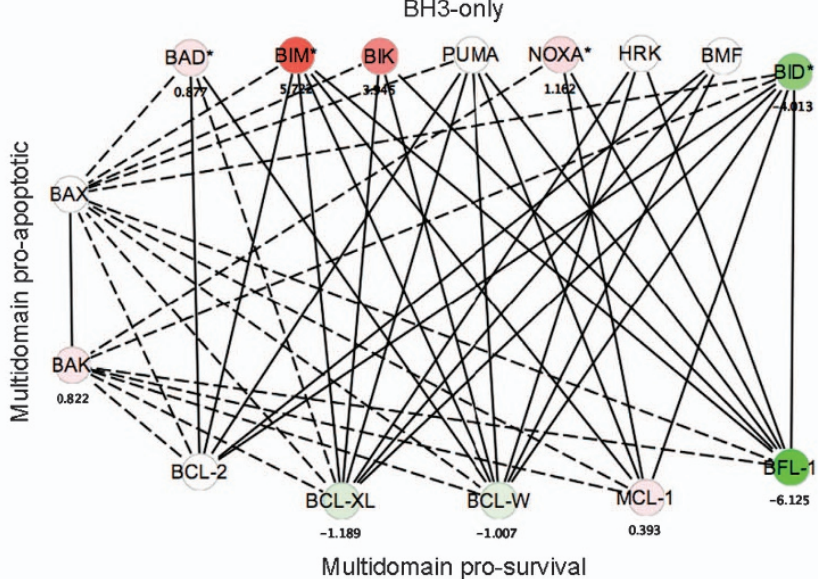

Figure 4 Ingenuity pathways analysis. Interaction map of the death receptor pathway was created using the ingenuity pathways analysis tool (a) BJ and (b) BJ LTSTERas fibroblasts. (c, d) An interaction map of the BCL-2 family of apoptotic regulators was created using the ingenuity pathways analysis tool, and overlaid with transcriptional responses to vorinostat in (c) BJ and (d) BJ LTSTERas fibroblasts. For (a-d), the direction and magnitude of vorinostat-mediated expression changes following $24 \mathrm{~h}$ of treatment, relative to time $\mathrm{O}$ h are indicated by pseudo-coloring. Induced (red), repressed (green) and non-responding probe sets (white) are shown. Log2 expression values of the strongest responding probe sets for each vorinostat-responding pathway component are shown. Interactions between BH3-only and pro-survival proteins are based upon Biacore analyses described in reference 26. Interactions between multi-domain proteins BAX/BAK and other BCL-2 family members were based on ingenuity pathway information

selectively induced in BJ LTSTERas cells. A candidate approach was subsequently undertaken to more thoroughly investigate the transcriptional regulation of apoptotic pathway components by vorinostat using the IPA program.

Vorinostat treatment resulted in decreased expression of TNFRSF6 (FAS), TNFRSF10A (DR4/TRAILR1) and TNFRSF1A (TNF $\alpha$ receptor) in both normal and transformed fibroblasts (Figures $4 a$ and b). Only a mild tumor-selective induction of TNFRSF10B (DR5/TRAILR2) was observed. We did not observe any transcriptional modulation of genes encoding death receptor ligands in response to vorinostat in either cell type (Figures $4 a$ and b). Moreover, the genes encoding FADD (FADD), caspase 8 (CASP8) and BID were downregulated in BJ and BJ LTSTERas, suggesting that death receptor signaling was transcriptionally repressed by vorinostat in BJ and BJ LTSTERas fibroblasts. Collectively, the downregulation of genes encoding receptors, adaptors and proteolytic intermediaries in the death receptor pathway suggested that the extrinsic apoptotic pathway was unlikely to play a substantial role in promoting selective apoptosis of BJ LTSTERas fibroblasts.

Vorinostat treatment caused the general transcriptional induction of the pro-apoptotic $\mathrm{BH} 3$-only protein encoding genes $B A D, B C L 2 L 11$ (BIM), BIK, PMAIP1 (NOXA), the multi-domain pro-apoptotic gene $B A K 1$ and genes encoding death effector components downstream of mitochondrial damage (DIABLO, APAF1, CASP9, HTRA2 and CYTC) in transformed fibroblasts. Moreover, the pro-survival genes BCL2A1, BCL2L1 (encoding $B C L-X L$ ) and BCL2L2 (encoding BCL-w) were concomitantly repressed in these cells (Figures $4 \mathrm{c}$ and $\mathrm{d}$ ). Collectively, this transcriptional response is predicted to confer a strong pro-death signal in the tumor cells.

Despite the downregulation of BCL2L1 and BCL2L2, normal BJ fibroblasts failed to mount a substantial proapoptotic transcriptional response. BIK, PMAIP1 and BAK1 probe sets were unchanged following vorinostat treatment, and $B A X$ and $B A D$ were repressed. Although the maximally responding probe set for BCL2L11 was induced in BJ and 
BJ LTSTERas cells (therefore, BIM is pseudo-colored red in Figures $4 \mathrm{c}$ and $\mathrm{d}$ ), three additional BCL2L11 probe sets were selectively induced in BJ LTSTERas cells (Supplementary Figure 2). Collectively, these data strongly support the hypothesis that the transformed cells were hypersensitive to vorinostat-mediated apoptosis due to acute transcriptional alterations in the balance of pro-death and pro-survival BCL2 family genes, whose protein products functionally converge to govern the integrity of the mitochondrial outer membrane.

Validation of BCL2 family gene expression by qRT-PCR. Expression of selected BCL2 family genes following vorinostat and dimethyl sulphoxide (DMSO) treatment was verified by qRT-PCR. qRT-PCR and microarray detection of vorinostat-induced transcriptional changes in $B A D, B A K 1, B I K, B C L 2 A 1$ were concordant (Figure 5a). $B C L 2 L 11$ (encoding BIM) was transcriptionally induced in normal and transformed BJ fibroblasts following vorinostat treatment, and BCL2L11 induction at the $4 \mathrm{~h}$ time point (by qRT-PCR) was consistently greater in the normal cells (Figure 5b). Seven Affymetrix probe sets map to BCL2L11. Interestingly, three of these probe sets responded to vorinostat in both cell types and three probe sets were selectively induced in BJ LTSTERas fibroblasts in a timedependent manner (Supplementary Figure 2). BMF was shown by us and others to be a primary HDACi response gene. ${ }^{27-29}$ In our microarray analyses, $B M F$ was classified as a non-responding gene most likely due to the combined effects of low basal expression and the location of the sole probe set (226530_at) toward the $5^{\prime}$ end of the BMF transcripts. The expression of BMF using qRT-PCR demonstrated that the gene was hyper-induced by vorinostat in BJ LTSTERas fibroblasts (Figure $5 b$ ). Taken together, the qRTPCR data supported the microarray-based observation of a a
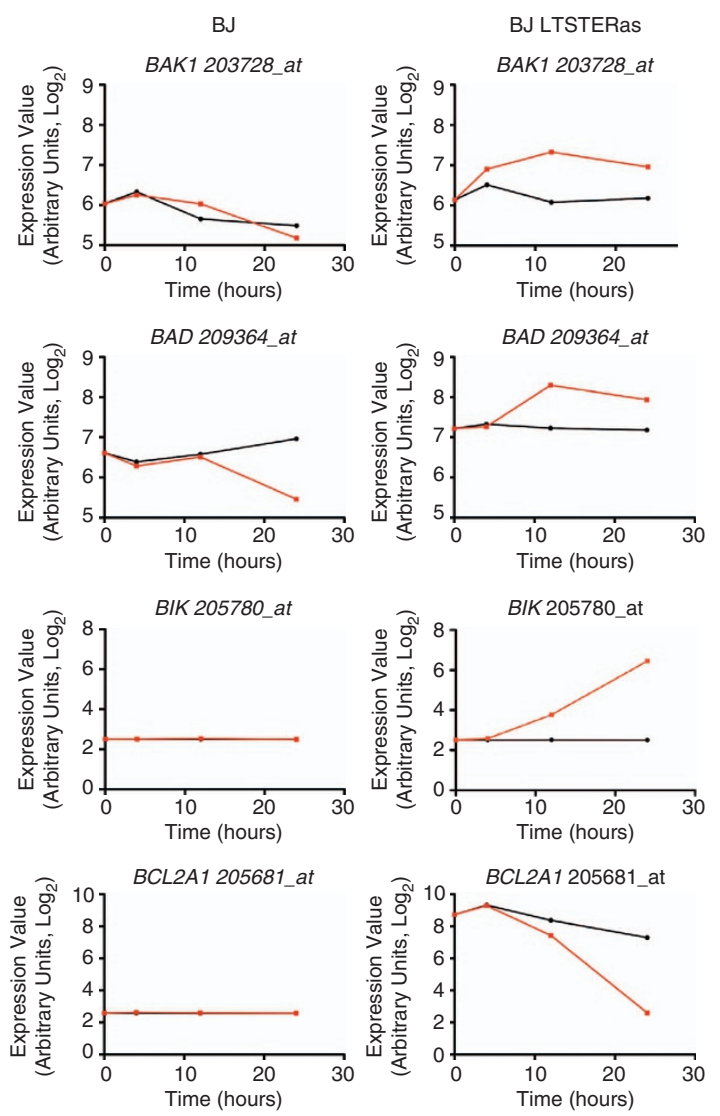

BJ LTSTERas
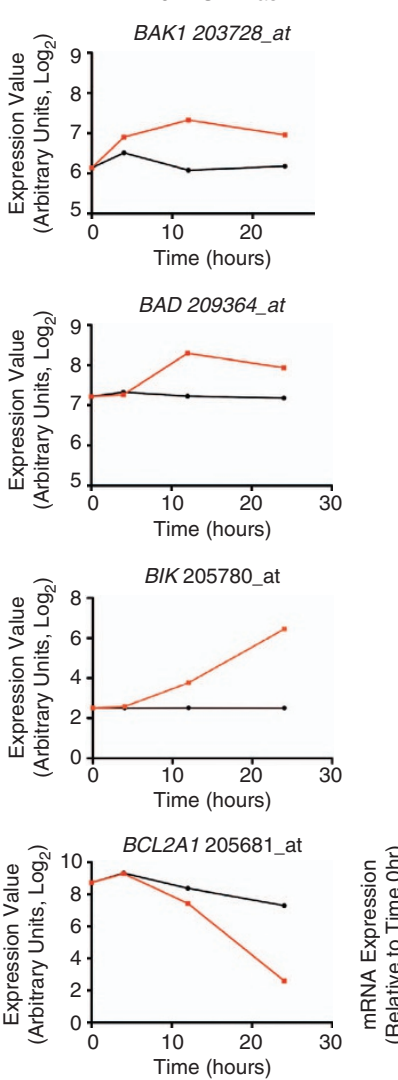

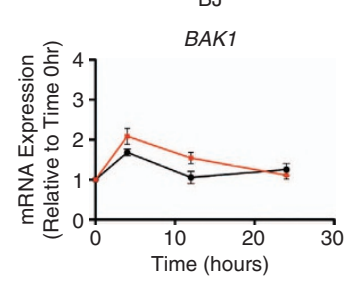

$B A D$
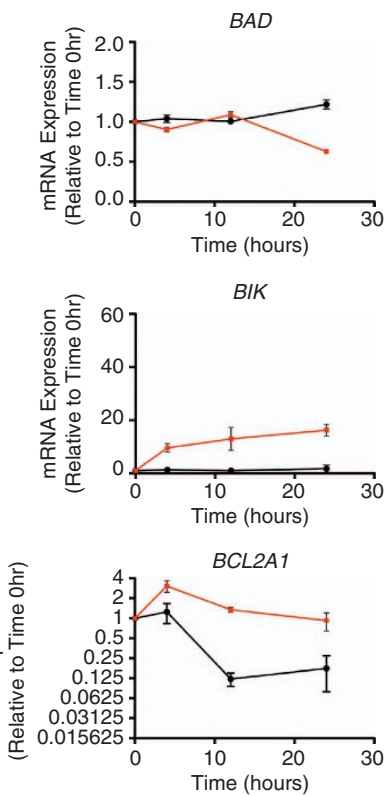

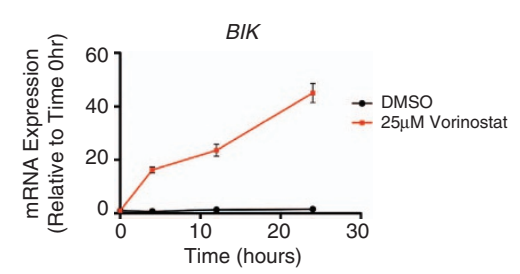

qRT-PCR
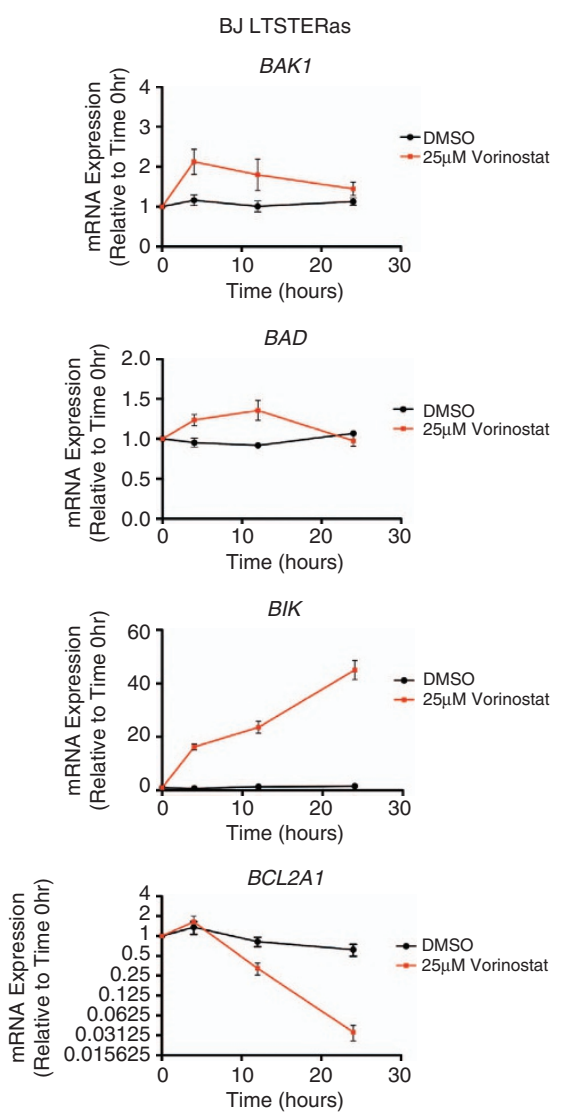

Figure 5 Validation of vorinostat-induced transcriptional responses by qRT-PCR. Transcriptional responses of BJ and BJ LTSTERas fibroblasts following DMSO (black) and $25 \mu \mathrm{M}$ vorinostat treatment (red) were validated by qRT-PCR using the same template RNA as that used in the microarray study. Log2 transformed expression intensities of HG U133 plus 2.0 probe sets corresponding to (a) BAK1,BAD, BIK and BCL2A1 and (b) BCL2L11 and BMF are shown. Gene names and probe set IDs are indicated in the title of each panel. Normalized probe set intensity values ( $Y$-axis) are presented, which allows direct comparison of basal expression (time $0 \mathrm{~h}$ ), and log2 transcriptional responses over time between cell types (BJ and BJ LTSTERas). Data represent the summarized probe set intensities from three independent microarray time course experiments. qRT-PCR verification of selected genes is shown. Transcript expression was calculated relative to the control gene RPL32 (that does not respond to vorinostat or DMSO treatment), and fold-change mRNA expression over time was calculated relative to the $0 \mathrm{~h}$ time point. Data are presented as the mean \pm S.E.M. of three independent experiments. For both Affymetrix and qRT-PCR results, data sets for BJ cells are in the left panels while data sets for BJ LTSTERas are in the right panels. (c) Whole cell protein extracts from BJ and BJ LTSTERas fibroblasts treated with DMSO or $25 \mu \mathrm{M}$ vorinostat for $0-48 \mathrm{~h}$ were separated by SDS-PAGE and analyzed for the expression of BMF, BIK and BIM by western blotting. The expression of $\alpha$-Tubulin was analyzed to confirm equal protein loading in each lane. The asterisk (*) denotes a non-specific band in the BIK western blot, which acts as a positive control for activity of the BIK antibody. The arrow denotes the location of BIK. Images are representative of two independent experiments 
b
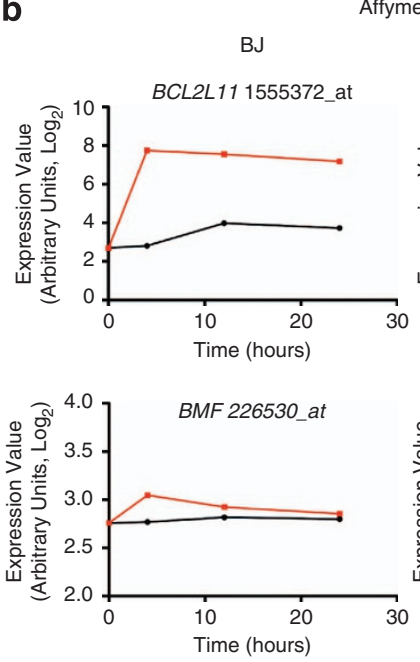

c
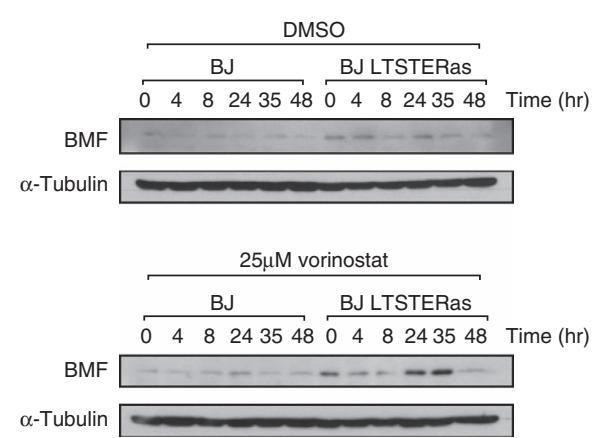

BJ LTSTERas
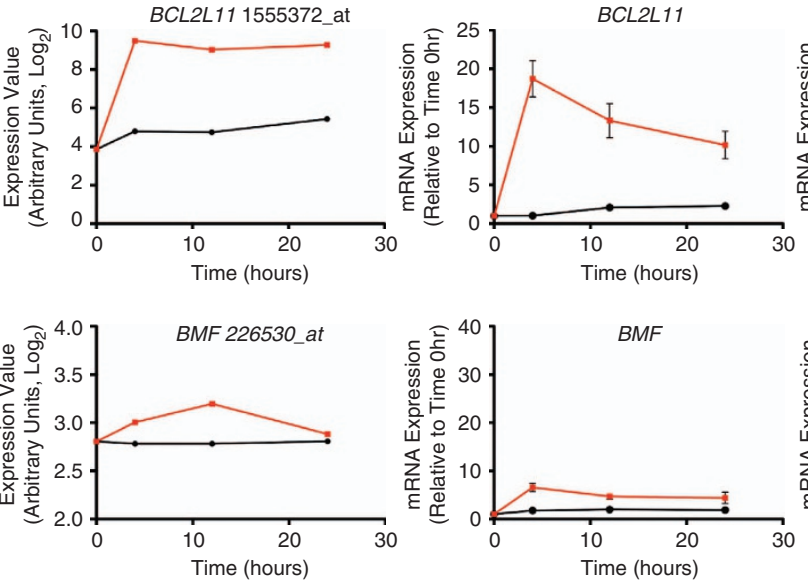

qRT-PCR
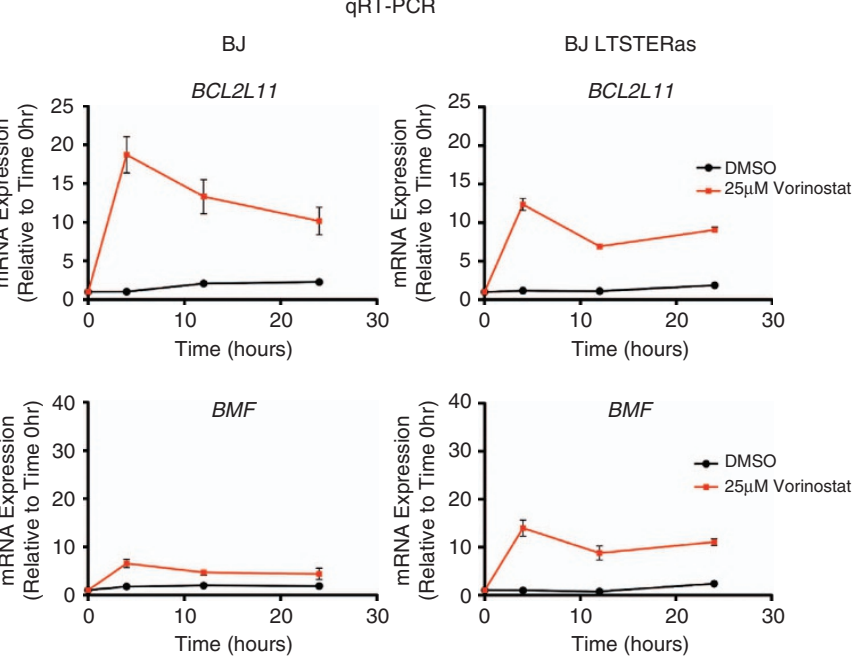

$\alpha$-Tubulin

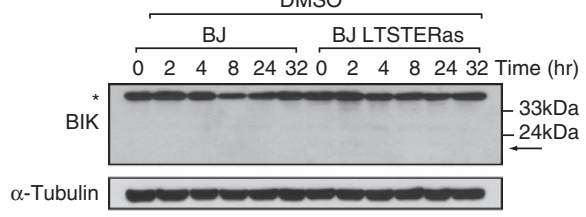

$25 \mu \mathrm{M}$ vorinostat

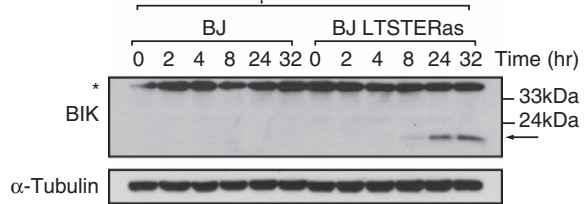

Figure 5 (Continued)

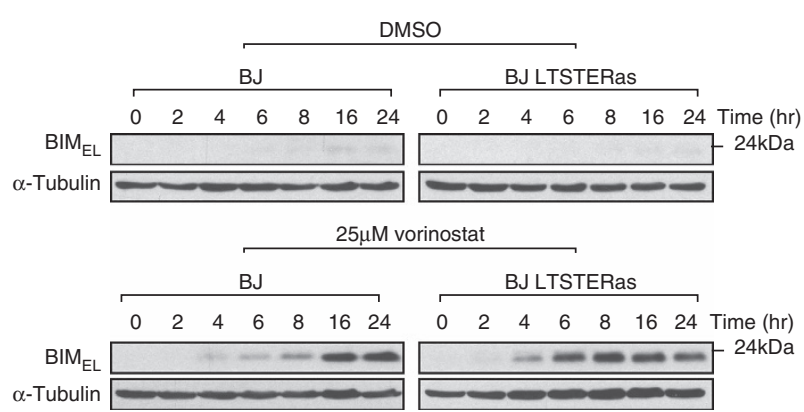

tumor-cell-selective pro-apoptotic gene expression signature, which temporally preceded the onset of apoptosis in BJ LTSTERas cells.

Western blotting was performed to confirm that the vorinostat-mediated transcriptional changes corresponded to alterated protein expression. BIK and BMF levels increased within $24 \mathrm{~h}$ of vorinostat treatment in BJ LTSTERas fibroblasts (Figure 5c). Despite the slight transcriptional induction of BMF and BIK mRNA in normal cells, changes in BIK and BMF protein levels were undetectable following vorinostat treatment in this cell type. $B M_{E L}$ protein levels were increased in BJ and BJ LTSTERas fibroblasts within $4 \mathrm{~h}$ of vorinostat treatment (Figure $5 \mathrm{c}$ ); however, the time to maximal $\mathrm{BIM}_{\mathrm{EL}}$ induction differed between the two cell types (16 h in BJ fibroblasts versus $6 \mathrm{~h}$ in BJ LTSTERas fibroblasts).

Vorinostat-mediated expression changes are HDACi-specific. To investigate whether vorinostatmediated changes in expression of Bcl-2-family genes were HDACi-specific, BJ and BJ LTSTERas cells were treated with equipotent concentrations of romidepsin or the DNA damaging agent etoposide and the expression of $B A K 1$, $B C L 2 L 11, B I K, B M F$ and BCL2A1 was analyzed by qRT-PCR (Figure 6). Similar to the response observed with vorinostat treatment, romidepsin induced $B C L 2 L 11$ in normal and transformed fibroblasts and induced $B A K 1$ in the transformed cells. Furthermore, romidepsin treatment 
caused the hyper-induction of $B I K$ and $B M F$, together with the marked repression of $B C L 2 A 1$ selectively in transformed cells. By contrast, etoposide failed to substantially induce $B M F$ or BIK. Furthermore, BCL2A1 was induced 22-fold (rather than repressed over time as observed with HDACi) in BJ LTSTERas fibroblasts by etoposide treatment. Etoposide was however clearly capable of modulating proapoptotic gene expression in matched fibroblasts, and caused the hyper-induction of BAK1 in BJ LTSTERas cells with a considerably higher magnitude than the changes induced by vorinostat or romidepsin. Collectively, these data strongly suggested that vorinostat-mediated alterations in the expression of $B C L 2$ family genes such as $B M F, B I K$ and $B C L 2 A 1$ were HDACi-specific.

\section{Forced expression of BCL2A1 protects BJ LTSTERas fibroblasts from vorinostat-mediated apoptosis.} $B C L 2 A 1$ was highly expressed in untreated BJ LTSTERas fibroblasts and demonstrated the strongest vorinostat response of all $B C L 2$ family genes with the pro-survival gene being strongly repressed in a tumor-cell-selective manner following HDACi treatment (refer to Figure 5a). BCL2A1 (encoding BFL-1) was over-expressed in BJ LTSTERas fibroblasts to ascertain whether restoration of $B C L 2 A 1$

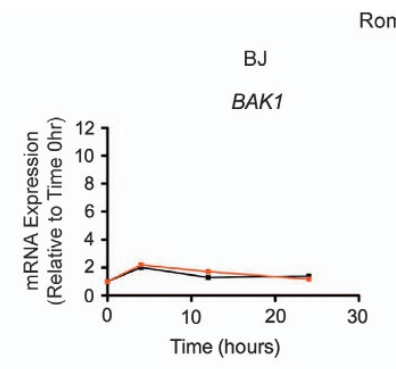

Romidepsin
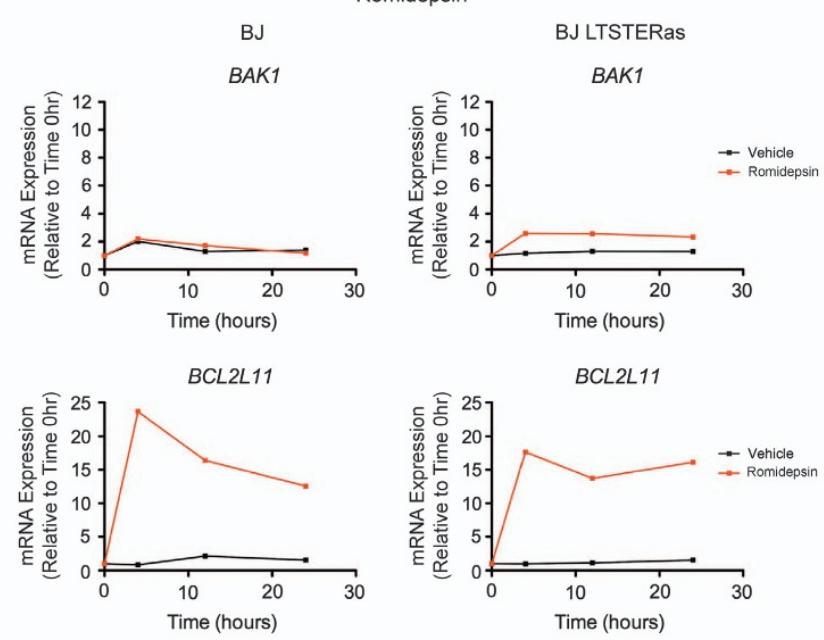

BIK

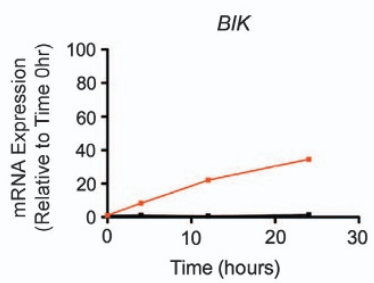

$B M F$

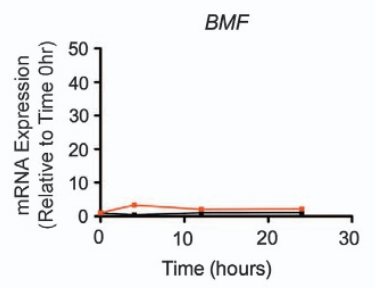

BCL2A1

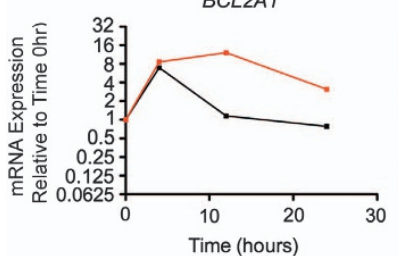

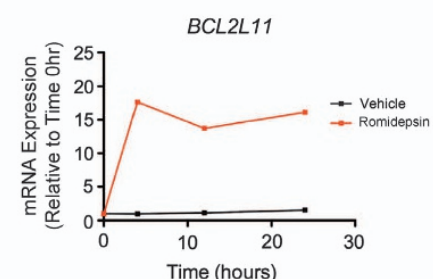

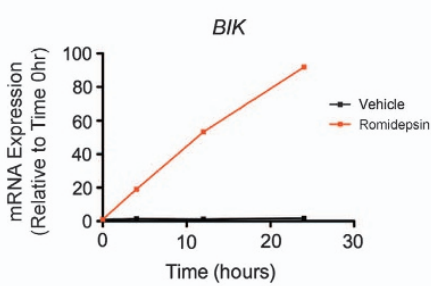

$B M F$

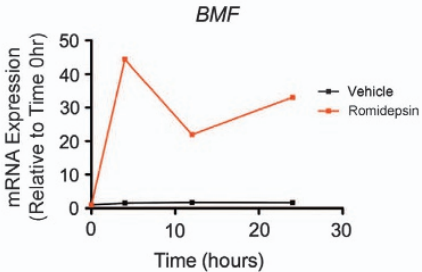

$B C L 2 A 1$

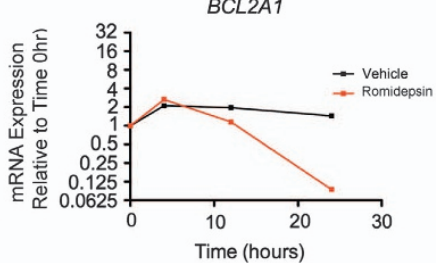

Etoposide
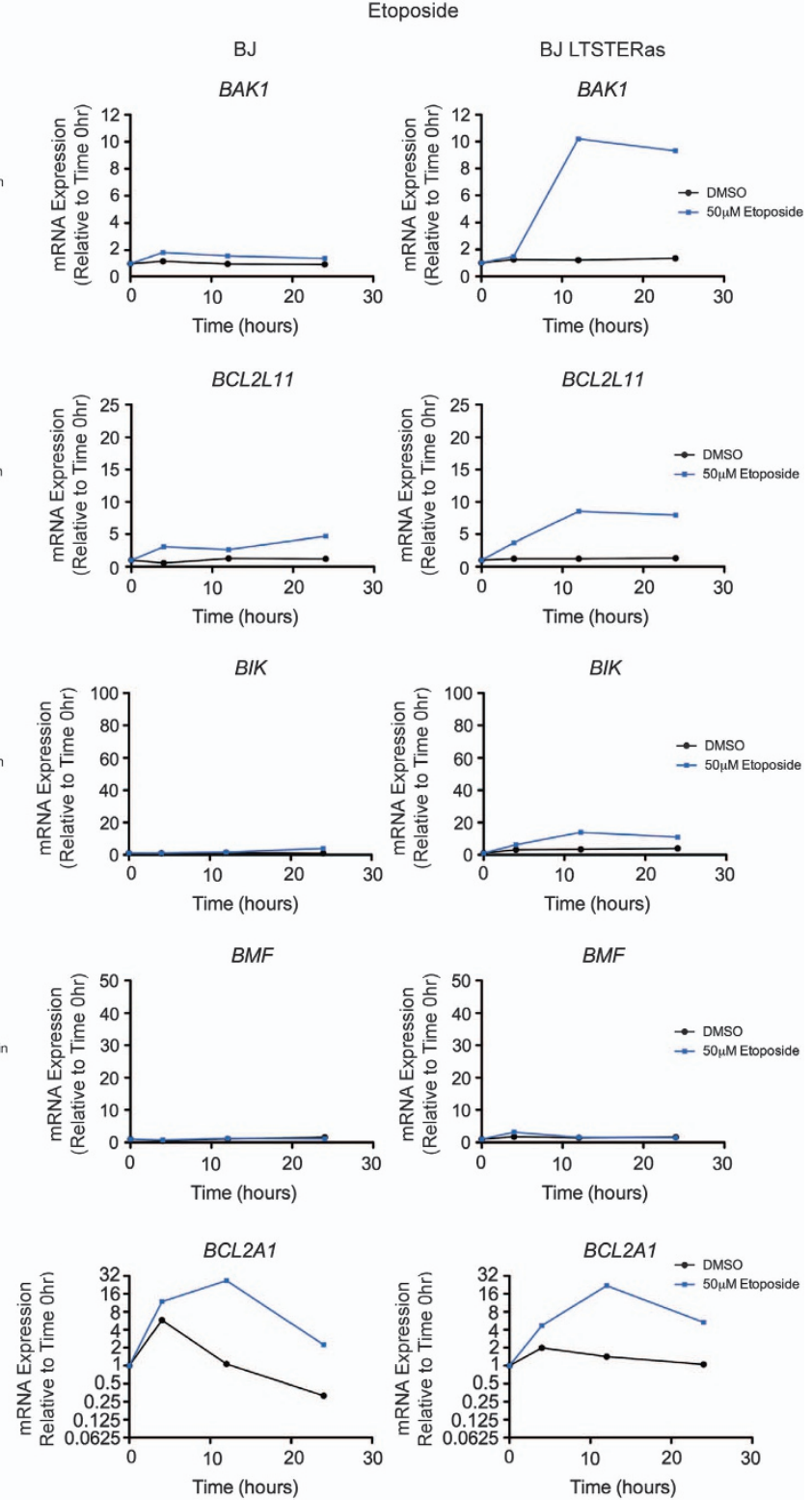

Figure 6 Regulation of $B C L 2$ family gene expression by the HDACi romidepsin and the DNA damaging agent etoposide. BJ (left panels) and BJ LTSTERas (right panels) fibroblasts were treated for $0,4,12$ and $24 \mathrm{~h}$ with $50 \mathrm{nM}$ romidepsin (red), $50 \mu \mathrm{M}$ etoposide (blue) and analyzed for the expression of BAK1, BCL2L11, BIK, BMF and BCL2A1 by qRT-PCR. Expression of each gene was calculated relative to the non-romidepsin- and non-etoposide-responding control gene RPL32 and fold-change expression over time was calculated relative to time $0 \mathrm{~h}$. Gene expression changes resulting from vehicle treatment (black) are also shown. Data are presented as the mean expression fold change from two independent time course experiments 
expression in BJ LTSTERas fibroblasts could protect cells from vorinostat-mediated death and to formally demonstrate that vorinostat-induced BJ LTSTERas cell death proceeds through induction of the intrinsic apoptotic cascade. BJ LTSTERas cells were transduced with an MSCVbased retroviral construct encoding FLAG-tagged human BFL-1 (FLAG-BFL-1), and expression of the exogenous FLAG-BFL-1 protein in GFP-positive cells was confirmed by western blot (Figure 7a).

The sensitivity of BJ LTSTERas cells transduced with MSCV empty vector or FLAG-BFL-1 to vorinostat-mediated apoptosis was evaluated alongside parental BJ LTSTERas and normal BJ fibroblasts. Expression of FLAG-BFL-1 significantly protected BJ LTSTERas fibroblasts from vorinostat-mediated apoptosis at the 48 and $72 \mathrm{~h}$ time points, relative to parental and MSCV transduced BJ LTSTERas fibroblasts (Figure $7 \mathrm{~b}, P<0.01$, as measured by two-way ANOVA at 48 and $72 \mathrm{~h}$ ). Collectively, these data showed that protection of cells at the level of mitochondrial integrity (through over-expression of a pro-survival BCL2 family gene) significantly attenuated the induction of vorinostat-mediated apoptosis in transformed fibroblasts, and therefore formally demonstrated a role for the intrinsic apoptotic cascade in the death of BJ LTSTERas fibroblasts.

\section{Discussion}

HDACi are being extensively used in the clinic as anti-cancer agents given their manageable toxicity profiles and broad antitumor activities. ${ }^{30}$ While tumor-cell-selective killing by HDACi has been observed, ${ }^{13,31-34}$ exactly why transformed cells are selectively hypersensitive to HDACi remains unclear. We aimed to determine whether donor-matched normal and transformed cells demonstrate the same or different gene expression profiles following HDACi treatment. Time-course microarray expression profiling revealed that normal and transformed cells transcriptionally responded to HDACi treatment, and that vorinostat signatures resembled other HDACi signatures in the Cmap database. Therefore, it appears that diverse HDACi modulate the expression
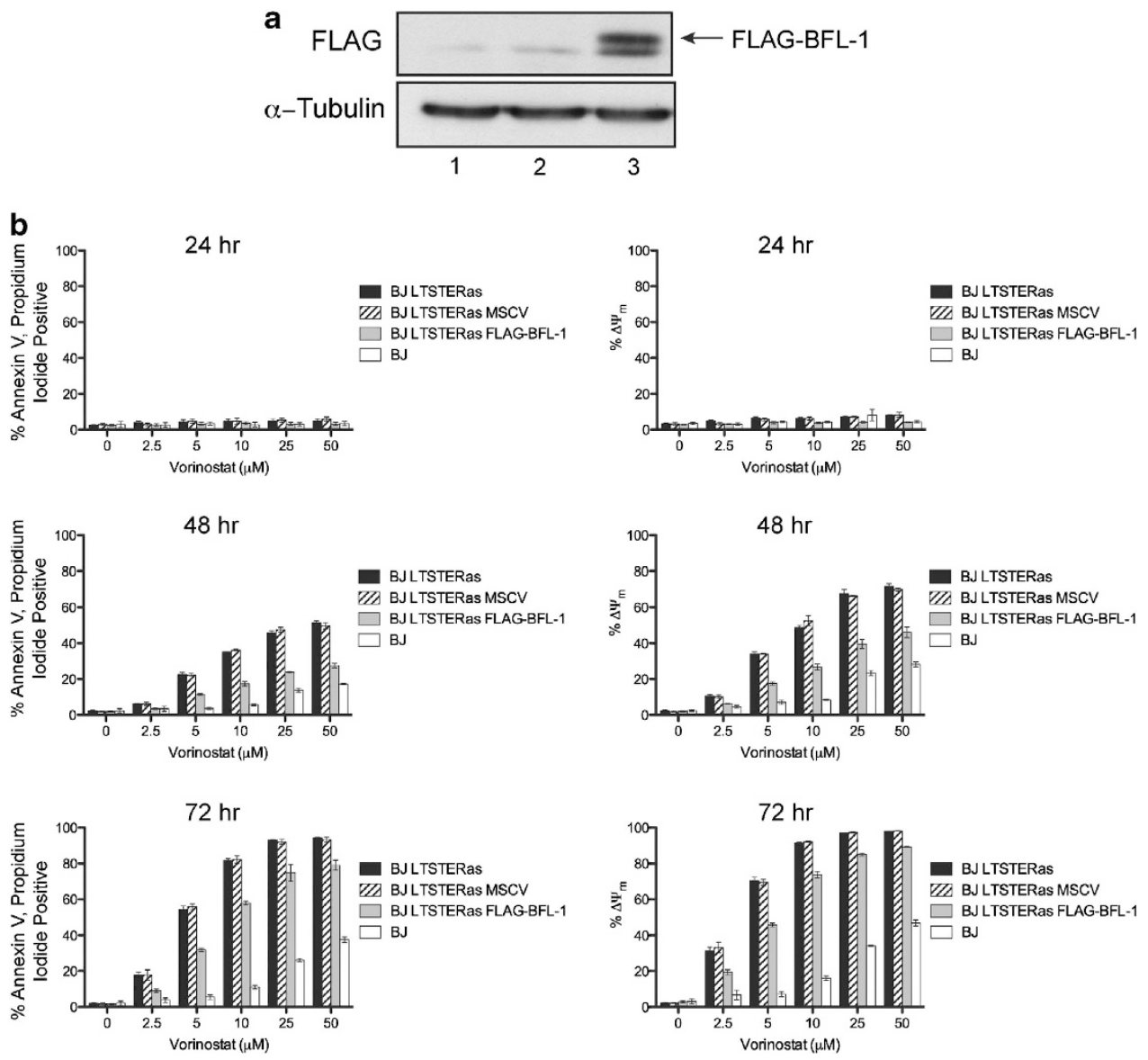

Figure 7 Forced expression of BFL-1 protects BJ LTSTERas fibroblasts from vorinostat-mediated apoptosis. (a) Proteins were extracted from exponentially growing BJ LTSTERas fibroblasts, and MSCV and MSCV FLAG-BFL-1 transduced variants. Proteins were separated on 10\% polyacrylamide gels by SDS-PAGE and analyzed for the expression of FLAG-BFL-1 by western blotting with an anti-FLAG antibody. The expression of $\alpha$-Tubulin levels served as a control for protein loading. (b) BJ, BJ LTSTERas, BJ LTSTERas MSCV and BJ LTSTERas FLAG-BFL-1 cells were incubated with 0, 2.5, 5, 10, 25 and $50 \mu \mathrm{M}$ vorinostat for 24,48 and $72 \mathrm{~h}$. Cells were harvested and stained with annexin-V-APC and propidium iodide as an apoptosis readout or stained for loss of mitochondrial outer membrane potential (MOMP, $\triangle \Psi \mathrm{m}$ ) using the mitochondrial dye tetramethylrhodamine ethyl ester (TMRE). Cells were then analyzed by flow cytometry. The percentage of annexin-V-APC, propidium iodide double positive cells are shown in the left panels while cells that had lost the ability to bind TMRE $(\Delta \Psi \mathrm{m})$ are in the right panels. Data are presented as mean \pm S.E.M. of three independent experiments 
of a common set of genes, irrespective of cellular phenotype or the tissues from which cells were originally isolated, and that a restricted set of genes are particularly amenable to transcriptional modulation following HDACi treatment. Many genes responded differently to vorinostat treatment in normal and transformed cells and a tumor-cell-selective pro-apoptotic gene-expression signature was identified. A role for HDACi-mediated repression of $B C L 2 A 1$ in the induction of tumor cell apoptosis was confirmed, and we formally demonstrated that the intrinsic apoptotic pathway was required for the cytotoxic activities of $\mathrm{HDACi}$ in transformed BJ fibroblasts.

A link between tumor-cell-selective gene induction and cell death following HDACi treatment was previously proposed. ${ }^{35,36}$ Kobayashi et al. analyzed romidepsin-mediated transcriptional responses in non-donor-matched normal melanocytes and melanoma cell lines and noted that RAP1A was selectively induced in melanoma cells, and was important for the cytotoxic effect of romidepsin in these cells. By contrast, we found RAP1A to be more highly induced in normal BJ fibroblasts than in BJ LTSTERas fibroblasts (data not shown), suggesting that any contribution of RAP1A towards HDACi-mediated cytotoxicity is likely to be celltype-dependent. Noh et al. ${ }^{36}$ investigated the transcriptional responses of non-donor-matched normal bronchial epithelium and lung cancer cells following TSA treatment. That study showed TSA mediated tumor-selective induction of $C D K N 1 A$ and tumor-selective repression of $E R B B 2,{ }^{36}$ both of which were also tumor-cell-selective events in our microarray study. Chiba et al. ${ }^{37}$ reported on the transcriptional responses of non-donor-matched hepatocytes and hepatocellular carcinoma cells. In agreement with our study, an approximately equal number of genes were activated and repressed in normal cells following HDACi treatment, although in our study a considerably higher percentage of total genes on the array were significantly modulated by vorinostat. ${ }^{38}$

Previous studies had proposed that altered ROS production and differential expression of ROS scavengers governs the differential sensitivity of normal and transformed cells to HDACi-mediated apoptosis. ${ }^{31-33}$ We did not observe any vorinostat-mediated transcriptional alteration in thioredoxin gene $(T X N)$ expression in normal or transformed cells. Moreover, the gene encoding TBP-2 (TXNIP) was equivalently induced with vorinostat treatment in both cell types. Therefore, vorinostat-mediated transcriptional modulation of TXN and TXNIP was unlikely to be a direct determinant of normal BJ fibroblast resistance, or transformed BJ fibroblast sensitivity to vorinostat-mediated apoptosis.

The most notable finding from our candidate and GO studies was the direction in which pro- and anti-apoptotic genes were transcriptionally altered in the normal and transformed cells. Vorinostat-treated BJ LTSTERas fibroblasts elicited a strong pro-apoptotic transcriptional response, consisting of the induction of pro-apoptotic BCL2-family genes and the concomitant repression of pro-survival genes. Although BCL2L1 and BCL2L2 were repressed in normal cells following vorinostat treatment, other pro-apoptotic genes such as $B A X, B A D$ and $B I D$ were also downregulated. Collectively, these data supported a model in which a tumorcell-selective alteration in the balance between pro- and antiapoptotic molecules following HDACi treatment ultimately determines the apoptotic sensitivity of cells exposed to these agents (Figure 8).

Previous studies have shown that HDACi can induce prodeath transcriptional signatures in human tumor lines. ${ }^{39-41}$ The regulation of $B C L 2$ family genes by HDACi in normal cells has to our knowledge not previously been investigated, making this study the first to characterize a

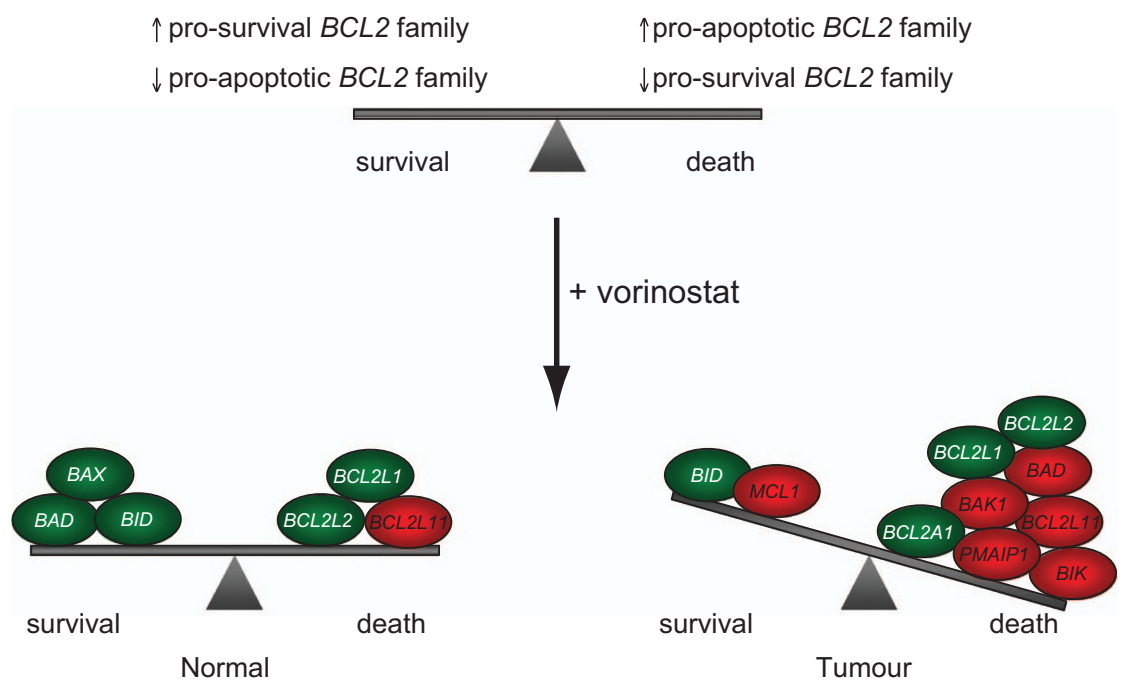

Figure 8 Model for the induction of tumor-selective apoptosis by vorinostat. The tumor-selective induction of apoptosis following vorinostat treatment may be due to acute alterations in the expression of pro-survival and pro-apoptotic BCL2 family genes. The vorinostat-mediated transcription changes observed in BJ LTSTERas cells are hypothesized to tip the balance of pro-survival and pro-apoptotic gene expression in a direction that favors cell death. Although pro-survival and pro-apoptotic expression changes were observed in normal cells, the net change of expression in terms of ability to 'tip the balance' is relatively neutral. Genes are pseudo-colored according to the direction of gene expression change at the $24-\mathrm{h}$ time point, relative to time $0 \mathrm{~h}$ : induced (red) and repressed (green), and are placed on the balance according to whether the expression change is expected to confer a pro-death or pro-survival response 
vorinostat-mediated BCL2 family transcriptional signature in donor-matched normal and transformed cells. The vorinostatregulated $B C L 2$ family genes identified herein (e.g., $B M F$, BCL2L11, PMAIP1, BCL2L1) have also been shown in independent studies to impinge upon HDACi-mediated apoptosis in tumor cells, ${ }^{16,27,42-45}$ suggesting that tumorcell-selective regulation of pro-apoptotic genes might be a common mechanism employed by HDACi to exert their tumorselective cytotoxic effect.

$B C L 2 L 11$ and BMF were rapidly induced early by vorinostat, indicating that they may be primary HDACi-response genes as has been reported in the literature for $B M F^{27}$ Despite the early transcriptional induction, an increase in BMF protein (selectively in tumor cells) was detected considerably later $(24 \mathrm{~h})$, and was consistent with BMF being a possible mediator of apoptosis in BJ LTSTERas fibroblasts. Previous reports suggested that elevated BIM expression alone (through deregulated E2F-1 activity) by HDACi was sufficient to induce apoptosis in E1A-expressing human fibroblasts. ${ }^{43,44}$ Moreover, BIM was reportedly important for the induction of apoptosis by HDACi in leukemic cell lines and primary cells from patients with chronic lymphocytic leukemia. ${ }^{42}$ We did observe vorinostat- and romidepsin-mediated induction of BCL2L11 mRNA by microarray and qRT-PCR in normal and transformed cells. Moreover, BIM $\mathrm{EL}_{\mathrm{L}}$ protein levels were elevated in both normal and transformed cells well before the onset of apoptosis (BIM induction was observed at $4 \mathrm{~h}$ ). These data were, therefore, inconsistent with BIM being the critical mediator of tumor-cell-selective apoptosis in the BJ fibroblast system and support the notion that BIM might instead cooperate with the many other HDACi-induced BH3only proteins (such as BMF and BIK) to induce apoptosis in sensitive cells.

Our data suggest that pro-death gene expression changes are likely to be important for the tumor-selective effects of HDACi; however, it is not yet known whether the HDACi signatures are driven by histone hyperacetylation in the promoters of specific loci and/or if gene modulation is due to the altered activities of transcription regulators. A recent report demonstrated a correlation between vorinotatmediated induction of BMF, NOXA and BCL2L11 and histone $\mathrm{H} 4$ hyperacetylation within the promoter regions of these genes following HDACi treatment. ${ }^{42}$ Whether such a correlation between HDACi-mediated selective induction of gene expression and promoter histone acetylation occurs on a global scale and is the basis for the tumor cell-specific effects of these anti-cancer agents remains to be determined.

\section{Materials and Methods}

Cell culture. BJ and BJ LTSTERas cells (kindly provided by Professor William Hahn, Dan Farber Cancer Institute, Boston, MA, USA) were grown in media containing four parts HEPES buffered DMEM, pH 7.4 (SAFC Biosciences, Brooklyn, Australia) to one part Medium 199 (Sigma, St. Louis, MO, USA), supplemented with $15 \%$ (v/v) fetal bovine serum (FBS; Commonwealth Serum Laboratories, Parkville, Australia), $1 \times$ GlutaMAX (Invitrogen, Carlsbad, CA, USA), $100 \mathrm{U} / \mathrm{ml}$ penicillin and $100 \mu \mathrm{g} / \mathrm{ml}$ streptomycin (Life Technologies, Inc., Grand Island, NY, USA). BMECs were cultured in Medium 199 (Biowhittaker, Walkersville, MD, USA) supplemented with $20 \% \mathrm{FBS}, 3 \%$ human serum, $20 \mu \mathrm{g} / \mathrm{ml}$ endothelial cell growth factor (Biomedical Technologies, Stroughton, MA, USA), $90 \mu \mathrm{g} / \mathrm{ml}$ sodium heparin (Sigma), $2 \mathrm{mM} \mathrm{L-Glutamine,} 80 \mathrm{U} / \mathrm{ml}$ penicillin and $80 \mu \mathrm{g} /$ $\mathrm{ml}$ streptomycin. The establishment of transformed BMEC (previously referred to as BMSVhTERT-4Nras) was previously described. ${ }^{19}$ Transformed BMEC were maintained in Iscove's Modified Dulbecco's Medium (IMDM; Life Technologies) with $20 \% \mathrm{FBS}$. All cells were maintained at $37^{\circ} \mathrm{C}$ in a humidified incubator containing $5 \% \mathrm{CO}_{2}$.

Chemical compounds. Vorinostat (100 mM) and $\mathrm{CHX}(5,50,500 \mu \mathrm{g} / \mathrm{ml})$ stock solutions were made in DMSO (Sigma) and stored at $-20^{\circ} \mathrm{C}$. Romidepsin was dissolved in $80 \%$ (v/v) 1,2 propanediol, $20 \%$ (v/v) ethanol by the Peter MacCallum Cancer Centre Pharmacy, and diluted to a $1 \mathrm{mM}$ stock concentration and stored at $-20^{\circ} \mathrm{C}$. Stocks were further diluted to working concentrations with growth media immediately before use. Cells were incubated with $0-50 \mu \mathrm{M}$ vorinostat for $0-72 \mathrm{~h}, 0-50 \mathrm{nM}$ romidepsin for $0-48 \mathrm{~h}$ and $50 \mu \mathrm{M}$ etoposide for $0-48 \mathrm{~h}$ to induce cell death and protein hyperacetylation. To inhibit new protein synthesis, cells were pre-treated (prior to the addition of HDACi) with $5,50,250$ or $500 \mathrm{ng} / \mathrm{ml} \mathrm{CHX}$ for $1 \mathrm{~h}$, and $\mathrm{CHX}$ was left in media throughout the $\mathrm{HDACi}$ treatment time course.

In vitro cell death analysis. BJ fibroblasts $\left(5 \times 10^{4}\right.$ cells) were seeded into wells of six-well plates (Greiner Bio-One, Stonehouse, UK). BMEC $\left(1 \times 10^{5}\right.$ cells) were seeded into $0.1 \%$ gelatin coated six-well plates. Cells were allowed to adhere overnight. Immediately prior to each experiment, spent media was replaced with fresh media and drugs/vehicles were added to working concentrations from stock solutions. At nominated time points, all floating and adherent cells (detached by trypsinization) were collected, washed with PBS, stained for viability markers and analyzed by flow cytometry. Mitochondrial outer-membrane permeability was measured with tetramethyl-rhodamine ethyl ester (TMRE-Molecular Probes, Eugene, OR, USA $)^{46}$ and DNA content determined by propidium iodide staining as previously described. ${ }^{16,17}$ Cell viability was measured by propidium iodide uptake and Annexin-V-APC staining. Briefly, cells were resuspended in annexin-V binding buffer ( $10 \mathrm{mM}$ Hepes pH 7.4, $140 \mathrm{mM} \mathrm{NaCl}, 5 \mathrm{mM} \mathrm{CaCl}$ ) containing 1/200 dilution of annexin-V-APC (BD Biosciences, San Jose, CA, USA) and 1/100 dilution of PI citrate buffer. Cells were analyzed using LSRII and FACSCanto II flow cytometers (BD Biosciences).

Western blotting. Cells $\left(1 \times 10^{6}\right)$ were seeded onto $10-\mathrm{cm}$ plates and incubated in the presence of $25 \mu \mathrm{M}$ vorinostat or DMSO. Cells were harvested by scraping into ice-cold PBS, washed once with PBS and lysed as described below. For analysis of BMF, BIK and BFL-1 cells were lysed in ice-cold whole-cell lysis buffer ( $150 \mathrm{mM} \mathrm{NaCl}, 50 \mathrm{mM}$ Tris-HCl pH 8.0, 0.1 mM EDTA, $0.1 \mathrm{mM}$ EGTA, $0.5 \%$ Triton X-100, 10\% glycerol) supplemented with protease inhibitors $(1 \mu \mathrm{g} / \mathrm{ml}$ Aprotinin, $1 \mu \mathrm{g} / \mathrm{ml}$ Leupeptin, $1 \mu \mathrm{g} / \mathrm{ml}$ Pepstatin and $2 \mathrm{mM}$ PMSF) for $1 \mathrm{~h}$, and the extracts cleared by centrifugation. For the analysis of acetylated proteins and BIM, cells were lysed on ice for $5 \mathrm{~min}$ with SDS lysis buffer $(250 \mathrm{mM} \mathrm{NaCl}, 50 \mathrm{mM}$ Tris$\mathrm{HCl}$ pH $7.5,5 \mathrm{mM}$ EDTA, $0.1 \%$ SDS, $1 \%$ Triton X-100, $1 \%$ sodium deoxycholate, $20 \%$ glycerol, $1 \mu \mathrm{g} / \mathrm{ml}$ Aprotinin, $1 \mu \mathrm{g} / \mathrm{ml}$ Leupeptin, $1 \mu \mathrm{g} / \mathrm{ml}$ Pepstatin and $2 \mathrm{mM}$ PMSF), sonicated twice for $15 \mathrm{~s}$ and extracts cleared by centrifugation. Proteins were separated on SDS-polyacrylamide gels and electroblotted onto Immobilon-P membranes (Millipore, Bedford, MA, USA). The following primary antibodies were used: anti-Acetylated tubulin (Clone 6-11 B-1, Sigma), anti-acetylated H3 (Upstate, Bellerica, MA, USA), anti- $\alpha$-tubulin (Clone B-5-1-2, Sigma) anti- $\beta$-Actin (Clone AC-74, Sigma), anti-BMF (Abcam, Cambridge, UK), anti-BIM (Stressgen, Farmingdale, NY, USA), anti-BIK (Santa Cruz, Dallas, TX, USA) and anti-FLAG (M2, Sigma). Membranes were incubated overnight with primary antibodies at $4{ }^{\circ} \mathrm{C}$, followed by short-term incubation with horseradish peroxidase (HRP)-conjugated secondary antibodies (DAKO) at room temperature. Immunoreactive bands were visualized by enhanced chemiluminescence (GE Healthcare, Little Chalfont, UK).

Quantitative real-time polymerase chain reaction. RNA was prepared from vehicle- and drug-treated cells $\left(1 \times 10^{6}\right)$ using $1 \mathrm{ml}$ TRIzol reagent according to the manufacturer's instructions (Invitrogen). RNA was resuspended in diethyl pyrocarbonate (DEPC)-treated, autoclaved $\mathrm{MQH}_{2} \mathrm{O}$ and first-strand cDNA was subsequently prepared using a random primer technique according to the manufacturer's instructions (Promega, Madison, WI, USA). Quantitative real-time PCR reactions were performed in triplicate using the SYBR green detection method in a 384-well plate format. Amplification and fluorescence detection was performed in a $7900 \mathrm{HT}$ fast real-time PCR system with SDS v2.3 software (Applied Biosystems, Grand Island, NY, USA). Initial denaturation of template and enzyme activation was performed at $95^{\circ} \mathrm{C}$ for $15 \mathrm{~min}$ followed by 40 cycles of $95^{\circ} \mathrm{C}$ for $30 \mathrm{~s}, 65^{\circ} \mathrm{C}$ for $30 \mathrm{~s}$. Dissociation protocols were always performed for each qRT-PCR reaction to ensure the presence of a single 'major' amplification product per well. An average $\mathrm{Ct}$ value for the three technical 
replicates was calculated for each sample. Fold-change expression of 'test' mRNA was determined relative to $R P L 32$ expression using the $2^{-\Delta \Delta C t}$ method. Expression fold-change over time was plotted relative to mRNA expression at the Oh time point.

\section{QRT-PCR primer sequences $\left(5^{\prime}-3^{\prime}\right)$}

RPL32. Forward TTCCTGGTCCACAACGTCAAG

Reverse TTGTGAGCGATCTCGGCAC

\section{CDKN1A. Forward GGCGGCAGACCAGCATGACAGATT Reverse GCAGGGGGCGGCCAGGGTAT}

\section{BCL2A1 (BFL-1). Forward CGTAGACACTGCCAGAACACTA Reverse GGGCAATTTGCTGTCGTAGA}

BAK1. Forward ACACTTGCTCCCAACCCATTCA Reverse AACCAAGTCCTAAGCCCTGT

BIK. Forward ATCTTGATGGAGACCCTCCTGT Reverse TCACTGCCCTCCATGCATT

\section{BMF. Forward ACCAGCCAGGAAGACAAAGCT Reverse CGATAGCCAGCATTGCCATAA}

\section{BAD. Forward AGTGACGAGTTTGTGGACTCCT Reverse AGCTTTGCCGCATCTGCGTT}

\section{BCL2L11 (BIM). Reverse GTTGCTTTGCCATTTGGTCT Forward CCACCACTTGATTCTTGCAG}

Microarray hybridizations. Total RNA $(2 \mu \mathrm{g})$ was prepared for hybridization to Affymetrix GeneChip Human Genome U133 Plus 2.0 Arrays according to the manufacturer's recommendations. Poly-A RNA controls from Bacillus subtilis (lys, phe, thr and dap) were diluted in Poly-A control dilution buffer (serial dilutions of $1: 20,1: 50$ and $1: 25$ ) and $2 \mu$ of the third serial dilution was spiked into each RNA sample prior to CDNA synthesis. RNA was reverse transcribed using a onecycle CDNA synthesis kit (Affymetrix, Santa Clara, CA, USA) and all annealing, amplification and incubation steps were performed in accordance with the manufacturer's instructions. Double-stranded CDNA was purified using a GeneChip sample cleanup module according to the manufacturer's instructions (Affymetrix) and cDNA was eluted from columns with $14 \mu \mathrm{l}$ cDNA elution buffer. Biotin-labeled cRNA was synthesized from cDNA using a GeneChip IVT labeling kit (Affymetrix) in a total reaction volume of $40 \mu \mathrm{l}$. CRNA was purified and concentrated using the GeneChip sample cleanup module. Full-length cRNA $(20 \mu \mathrm{g})$ was fragmented to $35-200 \mathrm{bp}$ lengths by incubation with cRNA fragmentation reagent for $35 \mathrm{~min}$ at $94^{\circ} \mathrm{C}$. Fragmented cRNA $(15 \mu \mathrm{g})$ was added to a hybridization mix according to Affymetrix instructions and hybridized to Affymetrix HG U133 plus 2.0 probe arrays at $45^{\circ} \mathrm{C}$ in an Affymetrix Hybridization oven 640 with 60 r.p.m. rotation for $16 \mathrm{~h}$. Array washes and three-stage streptavidin phycoerythrin (SAPE) staining was performed according to the manufacturer's instructions on a GeneChip fluidics station 450 (Affymetrix). HG U133 plus 2.0 arrays were individually scanned using an Affymetrix GeneChip 3000 scanner with GeneChip Operating Software (GCOS, Affymetrix).

Microarray differential expression analyses. Intensities from the Affymetrix arrays were background corrected, normalized and summarized using the GC-Robust Multi-Array Analysis (GC-RMA) algorithm. ${ }^{47}$ Probe sets were defined by the default Affymetrix HG U133 plus 2.0 chip definition file. Boxplots of expression intensities and RNA degradation plots confirmed good consistent quality of the samples and the hybridizations. Microarray data are available through the Gene Expression Omnibus, GEO accession number GSE43010

Differential expression analysis of the normalized log2-expression values was performed using the Bioconductor software package limma. ${ }^{48}$ All the arrays were analyzed together using a linear model. Empirical Bayes moderated $t$-tests were used to assess differential expression between the BJ and BJLTSTERas fibroblasts and between untreated and treated cells. ${ }^{48-50}$ Specifically, baseline differences between the cell lines were identified by comparing untreated $\mathrm{BJ}$ and to $\mathrm{BJ}$ LTSTERas fibroblasts at time $0 \mathrm{~h}$. Probe sets responding to DMSO or vorinostat were identified by comparing each of the 4,12 or $24 \mathrm{~h}$ time points back to untreated cells, and this was done separately for BJ and BJ LTSTERas fibroblasts. For all comparisons, probe sets were considered to be significantly differentially expressed if the fold-change exceeded 1.5 (i.e., $50 \%$ difference in expression) and the FDR was $<0.05^{51}$

Probe sets responding differently to vorinostat treatment in BJ LTSTERas fibroblasts as compared with BJ cells (i.e., contrast genes), were identified using empirical Bayes moderated F-tests. ${ }^{49,50}$ F-tests allowed us to combine information across the three treatment time points, looking for probe-sets with differing responses at any of the times. First we used an F-test to find probe sets whose gene expression changes (from $0 \mathrm{~h}$ to any of 4,12 or $24 \mathrm{~h}$ vorinostat treatment) were different in BJ fibroblasts compared with BJ LTSTERas fibroblasts. This test is analogous to testing for interaction in a two-way ANOVA. Second, we subtracted from this list any probe set whose expression response to vorinostat could not be distinguished from that to DMSO (the vehicle control). To do this, we used an F-test to find probe sets whose response to vorinostat relative to DMSO was different between BJ fibroblasts and BJ LTSTERas fibroblasts at any of the paired time points. Only probe sets detected by both the above F-tests were retained in the contrast list. The final contrast list contained therefore only bona fide vorinostatresponding genes. For all F-tests, probe sets were considered statistically significant if the FDR was $<0.05$ and at least one comparison involved in the test achieved a 1.5 -fold change.

GO analyses. Gene lists were tested for over-represented $\mathrm{GO}$ terms using the DAVID website. ${ }^{52}$ The Affymetrix HG U133 Plus 2.0 Array served as the background gene list. Only GO terms at specificity levels 4 or 5 within the directed acyclic graph hierarchy defined by the GO Consortium were included. ${ }^{53}$ The minimum hit threshold was set to 3 . Over-represented GO terms were ordered by the EASE score P-value, a conservative modification of Fisher's Exact test designed to de-prioritise annotation terms containing very small numbers of genes. ${ }^{54}$

Ingenuity pathway analyses. Gene lists and log2 expression fold-changes were uploaded into the IPA tool (Ingenuity Systems, www.ingenuity.com). When more than one probe set mapped to the same gene, the probe set with the largest fold-change was used. Pathways and interaction maps were constructed using the path designer function, with expression changes overlayed onto the pathways. Gene lists were also analyzed in IPA for overrepresented cellular and molecular functions and for association with disease. $P$-values were adjusted by the Benjamini and Hochberg algorithm to control the FDR at $<5 \%$.

Connectivity map analyses. Probe sets with statistically significantly (F-test) responses to vorinostat at any time were ranked by their expression fold-change at $24 \mathrm{~h}$ versus $0 \mathrm{~h}$, and this was performed separately for BJ and BJ LTSTERas cells. The 500 most induced and 500 most repressed probe sets were uploaded into the Connectivity Map (Cmap) v02 (http://www.broad.mit.edu/cmap). Cmap ranked drug compounds with gene expression signatures similar to the uploaded gene lists on the basis of connectivity $P$-values and enrichment scores. ${ }^{25}$ Cmap required Affymetrix HG U133A probe set identifiers, so the identifiers from our HG U133 Plus 2.0 Arrays were mapped back to equivalent HG 133A probe sets using the NetAffx analysis center (http://www.affymetrix.com/analysis/ index.affx).

Retroviral transduction of BJ LTSTERas fibroblasts. BFL-1 CDNA was cloned into the MSCV-IRES-GFP retroviral vector and transduction of BJ LSTERas cells was performed as previously described. ${ }^{16}$

\section{Conflict of Interest}

Dr. Johnstone's work has been funded by a research grant from Novartis.

Acknowledgements. We thank Ben Martin, Christopher Clarke and Richard Pearson for constructive criticism and helpful advice, and William Hahn for normal and transformed cell lines. RWJ is a Principal Research Fellow of the National Health and Medical Research Council of Australia (NHMRC) and supported by NHMRC Program and Project Grants, the Susan G Komen Breast Cancer Foundation, Cancer Council Victoria, The Victorian Cancer Agency, The Leukemia 
Foundation of Australia and the Victorian Breast Cancer Research Consortium. JEB was supported by The Cancer Research Institute Predoctoral Emphasis Pathway in Tumor Immunology. KLM was supported by funds from NHMRC, Cancer Institute NSW and NSW Cancer Council. GKS is a Senior Research Fellowship of the NHMRC. GKS and WS are supported by NHMRC Program Grant 490037.

1. Song J, Noh JH, Lee JH, Eun JW, Ahn YM, Kim SY et al. Increased expression of histone deacetylase 2 is found in human gastric cancer. APMIS 2005; 113: 264-268.

2. Choi JH, Kwon HJ, Yoon BI, Kim JH, Han SU, Joo HJ et al. Expression profile of histone deacetylase 1 in gastric cancer tissues. Jpn J Cancer Res 2001; 92: 1300-1304.

3. Krusche CA, Wulfing P, Kersting C, Vloet A, Bocker W, Kiesel L et al. Histone deacetylase1 and -3 protein expression in human breast cancer: a tissue microarray analysis. Breast Cancer Res Treat 2005; 90: 15-23.

4. Zhang $Z$, Yamashita $H$, Toyama $T$, Sugiura $H$, Omoto $Y$, Ando $Y$ et al. HDAC6 expression is correlated with better survival in breast cancer. Clin Cancer Res 2004; 10: 6962-6968.

5. Halkidou K, Gaughan L, Cook S, Leung HY, Neal DE, Robson CN. Upregulation and nuclear recruitment of HDAC1 in hormone refractory prostate cancer. Prostate 2004; 59 : 177-189.

6. Zhu P, Martin E, Mengwasser J, Schlag P, Janssen KP, Göttlicher M. Induction of HDAC2 expression upon loss of APC in colorectal tumorigenesis. Cancer Cell 2004; 5 : 455-463.

7. Wilson AJ, Byun DS, Popova N, Murray LB, L'Italien K, Sowa $Y$ et al. Histone deacetylase 3 (HDAC3) and other class I HDACs regulate colon cell maturation and p21 expression and are deregulated in human colon cancer. $J$ Biol Chem 2006; 281 13548-13558.

8. Spurling CC, Godman CA, Noonan EJ, Rasmussen TP, Rosenberg DW, Giardina C HDAC3 overexpression and colon cancer cell proliferation and differentiation. Mol Carcinog 2008; 47: 137-147.

9. Wu WS, Vallian S, Seto E, Yang WM, Edmondson D, Roth S et al. The growth suppressor PML represses transcription by functionally and physically interacting with histone deacetylases. Mol Cell Biol 2001; 21: 2259-2268.

10. Lin RJ, Nagy L, Inoue S, Shao W, Miller WH Jr., Evans RM. Role of the histone deacetylase complex in acute promyelocytic leukaemia. Nature 1998; 391: 811-814.

11. Ferrara FF, Fazi F, Bianchini A, Padula F, Gelmetti V, Minucci S et al. Histone deacetylasetargeted treatment restores retinoic acid signaling and differentiation in acute myeloid leukemia. Cancer Res 2001; 61: 2-7.

12. Gelmetti V, Zhang J, Fanelli M, Minucci S, Pelicci PG, Lazar MA. Aberrant recruitment of the nuclear receptor corepressor-histone deacetylase complex by the acute myeloid leukemia fusion partner ETO. Mol Cell Biol 1998; 18: 7185-7191.

13. Insinga A, Monestiroli S, Ronzoni S, Gelmetti V, Marchesi F, Viale A et al. Inhibitors of histone deacetylases induce tumor-selective apoptosis through activation of the death receptor pathway. Nat Med 2005; 11: 71-76.

14. Johnstone RW. Histone-deacetylase inhibitors: novel drugs for the treatment of cancer. Nat Rev Drug Discov 2002; 1: 287-299.

15. Minucci S, Pelicci PG. Histone deacetylase inhibitors and the promise of epigenetic (and more) treatments for cancer. Nat Rev Cancer 2006; 6: 38-51.

16. Lindemann RK, Newbold A, Whitecross KF, Cluse LA, Frew AJ, Ellis L et al. Analysis of the apoptotic and therapeutic activities of histone deacetylase inhibitors by using a mouse model of B cell lymphoma. Proc Natl Acad Sci USA 2007; 104: 8071-8076.

17. Newbold A, Lindemann RK, Cluse LA, Whitecross KF, Dear AE, Johnstone RW Characterisation of the novel apoptotic and therapeutic activities of the histone deacetylase inhibitor romidepsin. Mol Cancer Ther 2008; 7: 1066-1079.

18. Hahn WC, Counter CM, Lundberg AS, Beijersbergen RL, Brooks MW, Weinberg RA Creation of human tumour cells with defined genetic elements. Nature 1999; 400: 464-468.

19. MacKenzie KL, Franco S, Naiyer AJ, May C, Sadelain M, Rafii S et al. Multiple stages of malignant transformation of human endothelial cells modelled by co-expression of telomerase reverse transcriptase, SV40 T antigen and oncogenic N-ras. Oncogene 2002; 21: 4200-4211.

20. Bolden JE, Peart MJ, Johnstone RW. Anticancer activities of histone deacetylase inhibitors. Nat Rev Drug Discov 2006; 5: 769-784.

21. Grant C, Rahman F, Piekarz R, Peer C, Frye R, Robey RW et al. Romidepsin: a new therapy for cutaneous T-cell lymphoma and a potential therapy for solid tumors. Expert Rev Anticancer Ther 2010; 10: 997-1008.

22. Dickinson M, Johnstone RW, Prince HM. Histone deacetylase inhibitors: potential targets responsible for their anti-cancer effect. Invest New Drugs 2010; 28 (Suppl 1) S3-20.

23. Hahn WC, Dessain SK, Brooks MW, King JE, Elenbaas B, Sabatini DM et al. Enumeration of the simian virus 40 early region elements necessary for human cell transformation. Mol Cell Biol 2002; 22: 2111-2123.

24. Richon VM, Sandhoff TW, Rifkind RA, Marks PA. Histone deacetylase inhibitor selectively induces p21WAF1 expression and gene-associated histone acetylation. Proc Natl Acad Sci USA 2000; 97: 10014-10019.
25. Lamb J, Crawford ED, Peck D, Modell JW, Blat IC, Wrobel MJ et al. The Connectivity Map: using gene-expression signatures to connect small molecules, genes, and disease. Science 2006; 313: 1929-1935.

26. Chen L, Willis SN, Wei A, Smith BJ, Fletcher JI, Hinds MG et al. Differential targeting of prosurvival $\mathrm{Bcl}-2$ proteins by their $\mathrm{BH} 3-$ only ligands allows complementary apoptotic function. Mol Cell 2005; 17: 393-403.

27. Zhang $\mathrm{Y}$, Adachi M, Kawamura R, Imai K. Bmf is a possible mediator in histone deacetylase inhibitors FK228 and CBHA-induced apoptosis. Cell Death Differ 2006; 13 $129-140$.

28. Labi V, Grespi F, Baumgartner F, Villunger A. Targeting the Bcl-2-regulated apoptosis pathway by BH3 mimetics: a breakthrough in anticancer therapy? Cell Death Differ 2008; 15: 977-987.

29. Wiegmans AP, Alsop AE, Bots M, Cluse LA, Williams SP, Banks KM et al. Deciphering the molecular events necessary for synergistic tumor cell apoptosis mediated by the histone deacetylase inhibitor vorinostat and the BH3 mimetic ABT-737. Cancer Res 2011; 71 3603-3615.

30. Rasheed W, Bishton M, Johnstone RW, Prince HM. Histone deacetylase inhibitors in lymphoma and solid malignancies. Expert Rev Anticancer Ther 2008; 8: 413-432.

31. Butler LM, Zhou X, Xu WS, Scher HI, Rifkind RA, Marks PA et al. The histone deacetylase inhibitor SAHA arrests cancer cell growth, up-regulates thioredoxin-binding protein-2, and down-regulates thioredoxin. Proc Natl Acad Sci USA 2002; 99: 11700-11705.

32. Ungerstedt JS, Sowa Y, Xu WS, Shao Y, Dokmanovic M, Perez G et al. Role of thioredoxin in the response of normal and transformed cells to histone deacetylase inhibitors. Proc Natl Acad Sci USA 2005; 102: 673-678.

33. Xu W, Ngo L, Perez G, Dokmanovic M, Marks PA. Intrinsic apoptotic and thioredoxin pathways in human prostate cancer cell response to histone deacetylase inhibitor. Proc Natl Acad Sci USA 2006; 103: 15540-15545.

34. Chang J, Varghese DS, Gillam MC, Peyton M, Modi B, Schiltz RL et al. Differential response of cancer cells to HDAC inhibitors trichostatin A and depsipeptide. $\mathrm{Br} J$ Cancer 2012; 106: 116-125.

35. Kobayashi Y, Ohtsuki M, Murakami T, Kobayashi T, Sutheesophon K, Kitayama H et al. Histone deacetylase inhibitor FK228 suppresses the Ras-MAP kinase signaling pathway by upregulating Rap1 and induces apoptosis in malignant melanoma. Oncogene 2006; 25 512-524.

36. Noh EJ, Jang ER, Jeong G, Lee YM, Min CK, Lee JS. Methyl CpG-binding domain protein 3 mediates cancer-selective cytotoxicity by histone deacetylase inhibitors via differential transcriptional reprogramming in lung cancer cells. Cancer Res 2005; 65: 11400-11410.

37. Chiba T, Yokosuka O, Fukai K, Kojima H, Tada M, Arai M et al. Cell growth inhibition and gene expression induced by the histone deacetylase inhibitor, trichostatin $\mathrm{A}$, on human hepatoma cells. Oncology 2004; 66: 481-491.

38. Chiba T, Yokosuka O, Arai M, Tada M, Fukai K, Imazeki F et al. Identification of genes upregulated by histone deacetylase inhibition with CDNA microarray and exploration of epigenetic alterations on hepatoma cells. J Hepatol 2004; 41: 436-445.

39. Mitsiades CS, Mitsiades NS, McMullan CJ, Poulaki V, Shringarpure R, Hideshima T et al. Transcriptional signature of histone deacetylase inhibition in multiple myeloma: biological and clinical implications. Proc Natl Acad Sci USA 2004; 101: 540-545.

40. Peart MJ, Smyth G, Van Laar R, Bowtell D, Richon V, Marks PA et al. Identification and functional significance of genes regulated by structurally different histone deacetylase inhibitors. Proc Natl Acad Sci USA 2005; 102: 3697-3702.

41. Moore PS, Barbi S, Donadelli M, Costanzo C, Bassi C, Palmieri M et al. Gene expression profiling after treatment with the histone deacetylase inhibitor trichostatin A reveals altered expression of both pro- and anti-apoptotic genes in pancreatic adenocarcinoma cells. Biochim Biophys Acta 2004; 1693: 167-176.

42. Inoue S, Riley J, Gant TW, Dyer MJ, Cohen GM. Apoptosis induced by histone deacetylase inhibitors in leukemic cells is mediated by Bim and Noxa. Leukemia 2007; 21: 1773-1782.

43. Zhao Y, Tan J, Zhuang L, Jiang X, Liu ET, Yu Q. Inhibitors of histone deacetylases target the $\mathrm{Rb}-\mathrm{E} 2 \mathrm{~F} 1$ pathway for apoptosis induction through activation of proapoptotic protein Bim. Proc Natl Acad Sci USA 2005; 102: 16090-16095

44. Tan J, Zhuang L, Jiang X, Yang KK, Karuturi KM, Yu Q. Apoptosis signal-regulating kinase 1 is a direct target of E2F1 and contributes to histone deacetylase inhibitor- induced apoptosis through positive feedback regulation of E2F1 apoptotic activity. J Biol Chem 2006; 281: 10508-10515

45. Xargay-Torrent S, Lopez-Guerra M, Saborit-Villarroya I, Rosich L, Campo E, Roué G et al. Vorinostat-induced apoptosis in mantle cell lymphoma is mediated by acetylation of proapoptotic BH3-only gene promoters. Clin Cancer Res 2011; 17: 3956-3968.

46. Peart MJ, Tainton KM, Ruefli AA, Dear AE, Sedelies KA, O'Reilly LA et al. Novel mechanisms of apoptosis induced by histone deacetylase inhibitors. Cancer Res 2003; 63 $4460-4471$.

47. Wu Z, Irizarry RA, Gentleman R, Martinez-Murillo F, Spencer F. A model-based background adjustment for oligonucleotide expression arrays. J Am Stat Assoc 2004; 99 : 909-917.

48. Smyth GK. Limma: linear models for microarray data. In: Gentleman R, Carey V, Dudoit S, Irizarry R, H W (eds). Bioinformatics and Computational Biology Solutions using R and Bioconductor. Springer: New York, pp 397-420, 2005 
49. Smyth GK. Linear models and empirical bayes methods for assessing differential expression in microarray experiments. Stat Appl Genet Mol Biol 2004; 3, Article3.

50. Smyth GK, Ritchie M, Thorne N, Wettenhall J. limma: Linear Models for Microarray Data Users Guide 2005

51. Benjamini $Y$, Hochberg $Y$. Controlling false discovery rate: a practical and powerful approach to multiple testing. J Roy Statist Soc B 1995; 57: 289-300.

52. Dennis Jr G, Sherman BT, Hosack DA, Yang J, Gao W, Lane HC et al. DAVID: database for annotation, visualization, and integrated discovery. Genome Biol 2003; 4 R60.

53. Ashburner M, Ball CA, Blake JA, Botstein D, Butler H, Cherry JM et al. Gene ontology: tool for the unification of biology. The Gene Ontology Consortium. Nat Genet 2000; 25: 25-29
54. Hosack DA, Dennis Jr G, Sherman BT, Lane HC, Lempicki RA. Identifying biological themes within lists of genes with EASE. Genome Biol 2003; 4: R70.

Cell Death and Disease is an open-access journal published by Nature Publishing Group. This work is licensed under the Creative Commons Attribution-NonCommercial-No Derivative Works 3.0 Unported License. To view a copy of this license, visit http://creativecommons.org/licenses/by-nc-nd/3.0/

Supplementary Information accompanies the paper on Cell Death and Disease website (http://www.nature.com/cddis) 